\title{
A CROSSED BEAM APPARATUS FOR MEASURING DIFFERENTIAL CROSS SECTIONS FOR ION-NEUTRAL INTERACTIONS AT LOW COLLISION IENI:RGIIIS
}

\author{
James M. Farrar
}

September 1976

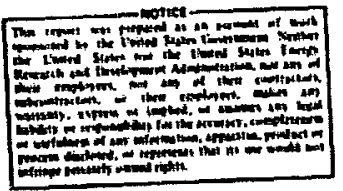


Tab1e of Contents

page

List of Illustrations. . . . . . . . . . . . . iv

Acknowledgements . . . . . . . . . . . . . . v

Introduction . . . . . . . . . . . . . . . . . 1

Chapter

I Discussion of Design Criteria. . . . . . . 8

II Detailed Description of Components . . . . 5

A. Main Chamber and Detection Chamber. . 15

B. Ion Beam Sources ar Focusing System . 21

1. Electron Impar: Source . . . . . 21

2. Microwave Discharge Source.... 26

3. Ion Beam Analysis and Focusing System ............ 27

Design Features. . . . . . . 35

operation. . . . . . . . . . 4

4. Beam Monitors. . . . . . . . 46

c. Molecular Beam Source. . . . . . . 46

D. Product Detection System . . . . . 53

1. Spherical Electrostatic Energy Analyzer

2. Read Lens Injection System .... 57

3. Heddle Lens Extraction System. . . 62

4. Quadrupole Mass Filter...... 69

E. Scintillation Ion Detector 70

1. General Remarks. . . . . . . . 70

2. EMI Detector.......... 75

3. RCA Detector.......... 76 
TABLE OF CONTENTS (continued)

Chapter

Page

II I Operation of the Apparatus . . . . . . . 82

IV Kinematic Analysis ........... 87

Appendix

A

Mechanical Drawing Index . . . . . . . 107

A. Molecular Beam Reaction Chamber. : . 107

B. Ion Beam Focusing System . . . . . 107

C. Electron Impact Ion Source..... . 108

D. Molecular Beam Source. . . . . . . . 108

E. Energy Analyzer. . . . . . . . . 109

F. Quadrupole Mass Spectrometer . . . . 109

G. Ion Detector . . . . . . . . . 110

H. Assembly Drawings. . . . . . . . 110

B Electronic Prints. . . . . . . . . . 111

A. Vacuum System............. 111

B. Experimental Instrumentation..... 111 


\section{List of I11ustrations}

Figure

Page

1 Block diagran of apparatus, top view. ....

Reaction chamber assembly... . . . . .

Electron impact ion source assembly . . . . . 23

Ion source mass spectrometer assembly . . .

Ion source mass spectrometer power supply block diagram. ............. 32

Initial focusing stage assembly ... . . . 34

Final focusing stage assembly . . . . . . .

8 Energy analyzer assembly. . . . . . . 65

9 Energy analyzer power supply block diagram. . 68

10 Scintillation detector assembly ...... 73

11 Velocity vector Newton diagram for a general crossed beam geometry. .........

12 Velocity vector Newton diagram showing construction of elenents of volume in the laboratory and c.m. coordinate systems..

13 Newton diagram showing derivation of Jacobian for a single recoil speed. . . . . . . 
Acknowledgements

It is a pleasure for the author to express his appreciation to the many individuals whose efforts brought the low energy ion molecule project to fruition. In particular, the author wishes to thank Professor Bruce H. Mahan, who provided him with financial support, advice of a philosophical and practical nature, and, most importantly, with patience, particularly when implementing many of the features of the apparatus required additional funds and time. The author has profited immensely under Professor Mahan's tutelage and wishes to thank him for committing two inportant years to the author's scientific development.

Thant:s are also due to Professor Yuan-tseh Lee, under whose guidance the author performed his graduate studies at The University of Chicago; Professor Lee's relocation in Berkeley has given the author an additional two years of profitable and enjoyable contact with him.

The services provided by the College of Chemistry machine shop have been vital in the completion of the project. Ir particular, the author acknowledges the contribution of Mr. Frank Lopez, whose skill and enthusiasm in fabricating many critical components in the apparatus has been instrumental in its successful operation. The author is also grateful to the College of Chemistry for providing some important funds for equipment purchases, especially for the ion pump on the detector. 
The operating procedures for this apparatus have been developed in collaboration with Mr. Steven Hansen, whose ability to grasp the principles of operation of the machine and to bring experiments to a successful conclusion has heen extremely important to the success of the project.

Finally, the author wishes to arknowledge the contributions of his most important collaborator and partner, his wife, Kathy. Her cheerful understanding of the demands of a research career and her presence and encouragement during times of success and failure have made an immeasureable contribution to the author's life, both professional and persona 1 . 
Introduction

The study of the rates at which chemical reactions proceed tas been a central area of research in chemistry for many years; in the last 20 years, the study of gas phase cnllision phenomena has been advanced by application of a number of sophisticated techniques. Spectroscopic techniques including the laser-induced fluorescence method pioneered by zare and co-workers ${ }^{1}$ have allowed the experimentalist to approach more closely the goal of deasurements of state-tostate reaction cross sections and one expects further advances in this field during the next several years. $\wedge$ second area of research in chemical kinetics, somewhat older than the first, is concerned with the preparation of the reactants by forming them into highly directional beams and observing the consequences of the collisions which occur when two reactant beams cross in a vacuum chamber. ${ }^{2}$ By appropriate experimental techaiques, the reaction products' mass and velocity can be determined, providing valuable information on the dynamics of the chemical reaction, that is, how the forces the molecules experience in a collision channel the total energy of the system into product translational and internal degrees of freedom. In principle, the study of chemical reactions under well-defined conditions of initial relative velocity in a single-collision environment should provide intimate details about the various interactions between approaching reactants and departing products. The molecular beam technique is especially suited to such high resolution studies of chemical reactions and 
significant effort has been expended in recent years to extend the scope of beam studies of chemical kinetics. For reactions of transient species, meaningful studies of chemical reactions almost require that a beam technique be used for the preparation of isolated reactants and detection of products. The study of the chemical reactions of gaseous ions provides one with examples of systems in which beam techniques have been quite successful in elucidating reaction dynamics. ${ }^{3}$ Indeed, mass spectrometers have always employed beam techniques, exploiting the focusing and analyzing capabilities of electric and magnetic fields. Many of the results which have been obtained in the past have involved conventional mass spectrometers and, in fact, the first ion-molecuie reaction observed in a inass spectrometer, $\mathrm{H}_{2}{ }^{+}+\mathrm{H}_{2} \rightarrow \mathrm{H}_{3}{ }^{+}+\mathrm{H}$, was observed at high source pressures quite by accident in 1912 when $J$. J. Thomson noted a mass 3 signal upon admitting hydrogen gas to his apparatus. It was not until 1925, however, that Hogness and Lund explained Thomson's observation by direct observation of the above ion-molecule reaction.

As experimental advances have occurred, beam techniques have become more sophisticated and significant improvements in beam experimentation on ion-molecule collision dynamics have taken place in the last 10 years. In this laboratory, an ion beam apparatus has been in operation for several years, ${ }^{4}$ employing an ion beam plus a scattering gas cell; this device has been used successfully for a number of experimental studies in the laboratory energy range 20-250 eV. This 
venerable apparatus has been used for several years with great success and the data obtained with it have been the nucleus of many Ph.D. theses. Many ion-molecule reactions in the high-energy regime have been studied and elegant but understandable models have been developed to explain these results. However, the relative energy range accessible to this instrument has a lower limit near $2 \mathrm{eV}$, above the bond energies of many species. Clearly, the lower energy regime, inaccessible to this instrument, should be of great chemical interest as one moves away from the range where collisions can he discussed in terms of impulsive, hard-sphere interactions. About the $i$ ime this apparatus was constructed, the ion beam apparatus fabricated by Herman and Wolfgang became operational. 5 This apparatus employed a momentum analyzer for primary bean production, a multichannel array neutral crossed bean and a retarding field energy analyzer for the product ions. This apparatus could be used down to laboratory energies of $0.6 \mathrm{eV}$ but its versatility has been limited by its poor vacuum system and the lack of sensitivity inherent in integral energy analyzers.

Two very new machines are now in operation with the explicit goal of examining the dynamics of low energy ionmolecule reactions: the first, by Futrel1 and co-workers 6 employs a chemical ionization ion source followed by a momentum analyzer. A crossed nozzle beam provides the second beam; differential cross sections of reactively scattered products are detected by a plane field retarder followed by a mas filter, although recent work has been performed with a $90^{\circ}$ 
cylindrical electrostatic analyzer. Extensive work has been performed on the reactions of $\mathrm{H}_{3}{ }^{+}$which is produced in the chemical ionization source. The major drawback of this apparatus involves the limited number of ions which can be produced by chemical ionization.

The second new machine constructed for low energy studies is that of Koski et al. ${ }^{7}$ This apparatus produces an energy and mass selected ion beam by use of an electron impact source followed by a $180^{\circ}$ spherical electrostatic energy analyzer in tandem with a quadrupole mass filter. The beam then enters a scattering s,as cell and product ions are energy and mass analyzed by another $180^{\circ}$ deflector - quadrupole mass spectrometer combination. A consideration of the velocity distribution of the neutral species in the scattering cell suggests immediately that the distribution of initial relative velocity vectors can be quite broad for low energy experiments; for example, an $\mathrm{F}^{+}$ion with $1 \mathrm{eV}$ energy has a speed of $3.2 \mathrm{x}$ $10^{5} \mathrm{~cm} / \mathrm{sec}$. A $\mathrm{D}_{2}$ molecule at $300^{\circ} \mathrm{K}$ has a most probable speed of $1.1 \times 10^{5} \mathrm{~cm} / \mathrm{sec}$; in a scattering cell, the distribution of $\mathrm{D}_{2}$ velocities is isotropic, so that the initial relative velocity vector distribution is prohibitively broad. Consequent1y, even with monoenergetic low-energy ion beams, the spread in initial conditions brought about by use of a scattering cell is too large to make results obtained at the lowest energies reliable.

In order to initiate a program of scattering studies of low energy ion-molecule reaction dynamics in this laboratory, we have fabricated a new apparatus which we discuss in detail 
in this report. This apparatus was designed and constructed with the following criteria in mind:

1. Production of intense (space-charge limited) ion beams of a wide variety of species at low laboratory energies (1-10 ev) with narrow energy distributions.

2. The machine should embody the "fixed source movable detector" geometry to accommodate the differential pumping required for a supersonic nozzle crossed beam.

3. Cleanliness to reduce the buildup of insulating layers which will "charge up" under ion impact and deflect low energy ions.

4. The detector chamber should be valved so that it can remain under vacuum while sources are repaired or replaced as required.

5. The detector should be provided with differential pumping to keep the background pressure low, thereby suppressing large cross section, low energy secondary ion-molecule reactions with background gas in the detector.

6. A supersonic nozzle beam source should be employed as a neutral source to avoid the poorly defined initial conditions brought about by use of a scattering cell at low collision energies.

The appuratus to be described here embodies these six criteria; the sixth point has not been fully implemented at the time this report is being prepared, but a rudimentary. supersonic molecular beam source has been used successfully. 
This report will describe the apparatus as it stands currently and future modifications will be discussed extensively.

The apparatus has been used to study the systems $\mathrm{N}^{+}\left(\mathrm{H}_{2}, \mathrm{H}\right) \mathrm{NH}^{+}$and $\mathrm{N}^{+}\left(\mathrm{H}_{2}, \mathrm{H}_{2}\right) \mathrm{NH}^{+}$for which extensive data have been collected at higher relative energies and for which some recent ab initio potential energy surface calculations suggest that low energy experiments might be instructive. We have also recently begun the systems $\mathrm{F}^{+}\left(\mathrm{H}_{2}, \mathrm{H}\right) \mathrm{FH}^{+}$and $\mathrm{F}^{+}\left(\mathrm{H}_{2}, \mathrm{H}_{2}\right) \mathrm{I:}^{+}$ for which some datis by Koski et al. are available. 
References, Introduction

1. A. Schultz, H. W. Cruse, and R. N. Zare, J. Chem. Phys. 57, 1354 (1972).

2. K. P. Lawley, ed., Advances in Chemical Physics, Vo1. 30, Molecular Beam Scattering (Interscience, New York, 1975).

3. See, for example, J. L. Franklin, ed., Ion-Molecule Reactions, Vol. I, II (Plenum, New York, 1972).

4. W. R. Gentry, University of California, Lawrence Radiation Laboratory Report UCRL-17691 (1967).

5. 2. Herman, J. D. Kerstetter, T. L. Rose, and R. Wolfgang, Rev. Sci. Instrum. 40, 538 (1969).

6. M. L. Vestal, C. R. Blakley, P. W. Ryan, and J. H. Futrell, Rev. Sci. Instrum. 47, 15 (1976).

7. K. Wende11, C. A. Jones, J. J. Kaufman, and W. S. Koski, J. Chem. Phys. 63, 750 (1975). 
CHAPTER I

\section{Discussion of Design Criteria}

The performance of successful molecular beam scattering experiments requires attention to detail and an understanding of the limitations and difficulties associated with detecting product species ac low levels. The detection of low product ion fluxes in the range of one ion $/ \mathrm{cm}^{2} \mathrm{sec}$ is one which has generally been solved as Gentry ${ }^{1}$ has indicated, but one should note that such detection efficiency can be achieved only when stray fields do not prevent products from reaching the detector. While ions with energies above $20 \mathrm{eV}$ will not be influenced strongly by such stray fields, low energy ions near 1 eV can only be detected by paying special attention to these extraneous fields. Further difficulties which are encountered in the production of low energy ions relate to the mutual Coulomb interaction of the ions, or space charge, which limits the beam current density achievable without spreading of the beam. ${ }^{2}$ Consequently, high intensity, well-collimated beams of low energy ions are very difficult to produce and detect. In the Introduction to this work, we have identified some of the design criteria which were established as solutions to these and other problems and we now discuss the points in groater depth.

The production of space charge limited beams of low cnergy ions consistent with narrow energy and angular spread is a problem attached successfully by a few groups. In the absence 
of energy analysis of the primary ion beam, the energy spread of the source is determined essentially by the electric field distribution in the region of space where the ions are formed. This statement is incomplete, however, if one does not consider the possibility that the ionization process imparts additional kinetic energy to the ions, one expects this to occur when ions are produced as a result of dissociative ionization of a parent molecule. For example, in our studies of $\mathrm{N}^{+}$scattering, we have measured FWHM energy spreads as large as $1 \mathrm{eV}$ for $\mathrm{N}^{+}$ ions produced by electron impact on $\mathrm{N}_{2}$, whereas the corresponding $\Delta \mathrm{E}$ for $\mathrm{N}_{2}{ }^{+}$has been determined to be as low as $0.2 \mathrm{eV}$ under similar conditions. While energy analysis of the primary ion beam can be accomplished, the increase in apparatus complexity is significant and we have chosen instead to optimize our ion source. A detailed iescription of the source is found later in this report.

The "fixed source-movable detector" geometry has been embodied to allow for the substantial differential pumping required to implement supersonic nozzle sources. 3 Such an arrangement results in a much better defined velocity distribution contributing to higher resolution experiments at low collision energies. The original configuration for the scattering chamber precludes a separate differential pumping chanver for the neutral beam source although a cylindrical chamber concentric to the nozzle heam formation chamber has been designed to overcome this problem to a certain extent. The initial solution to this problem involved use of a non= differentially pumped supersonic nozzle in conjunction with a 
conical "beam catcher" mounted opposite to the beam source and pumped out through the apparatus sidearm.

cleanliness in the ion source and detector is a continuing problem but satisfactory solutions have been achieved; the diffusion pumps for the apparatus are trapped with liquid nitrogen cooled baffles which prevent the silicone pump oil (DC 704) from condensing on the optics. While this procedure is reasonably efficient, we find that the initial focusing stage optics require cleaning at intervals as frequent as every two weeks. Although the optics are coated with colloidal graphite (Aquadag), no additional solution to this problem has been found aside from regular cleaning.

The problem of cleanliness in the detection chamber has been attacked by noting that the predominant difficulty arises from buildup of surface charge on the detector entrance slit. The problem is cumulative and without precautions, the transmission of low energy ions through the detector is impaired by two or three orders of magnitude. A satisfactory solution to this problem is achieved by heating the front slit of the detector above $100^{\circ} \mathrm{C}$ with a non-inductivity wound cartridge heater. The detector surfaces are then heated to the extent that adsorption of insulating materials is strongly inhibited. An increase in transmission of one thousand for $1 \mathrm{eV} \mathrm{N}{ }_{2}^{+}$ions was noted immediately after this modification to the apparatus. The detection thamber for this apparatus is vilvahle from the exterior of the vacuum chanber; this feature allows one to maintain vacuum in the detector while performing maintenance on the bean sources of the apparatus as required. Because of 
this feature, it becomes feasible to incorporate differcntial pumping with an efficient ion pump in the detector design with two clear advantages:

1. The clean vacuum associated with such a pump should lead to very little required cleaning of detector components .

2. Differential pumping of the detection chamber reduces the background pressure level, thereby diminishing spurious signal arising from large cross-section ion-molecule reactions at low energies.

Both of these points are of extreme importance in the low energy regime; increased detector complexity from the ion optics required to inject low energy ions into the energy analyzer results in more elements which must be kept extremely clean. Reactions of background gas with thermal ions can proceed with cross sections as large as those predicted by the Langevin model and can obliterate true reactive events if not suppressed.

In succeeding chapters of this report, the detailed design criteria for this apparatus will be discussed. A top view of the apparatus indicating schematically the placement of the various components is shown in Figure 1. Our experience with this apparatus to date suggests that the six criteria we have discussed for low energy machines of this type are sufficient (and probably necessary) to make the chemically interesting regime of low energies accessible to crossed beam studies. 


\section{References, Chapter I}

1. W. R. Gentry, University of California, Lawrence Radiation Laboratory Report UCRL-17691 (1967).

2. J. R. Pierce, Theory and Design of Electron Beams, 2nd Ed. (van Nostrand, New York, 1954).

3. J. B. Anderson, R. P. Andres, and J. B. Fenn, Adv. Chem. Phys. 10, 289 (1966). 
Figure 1. Block diagram of the source and detector components of the apparatus shown in a top view. The detector is shown at a positive laboratory angle $\theta$. 


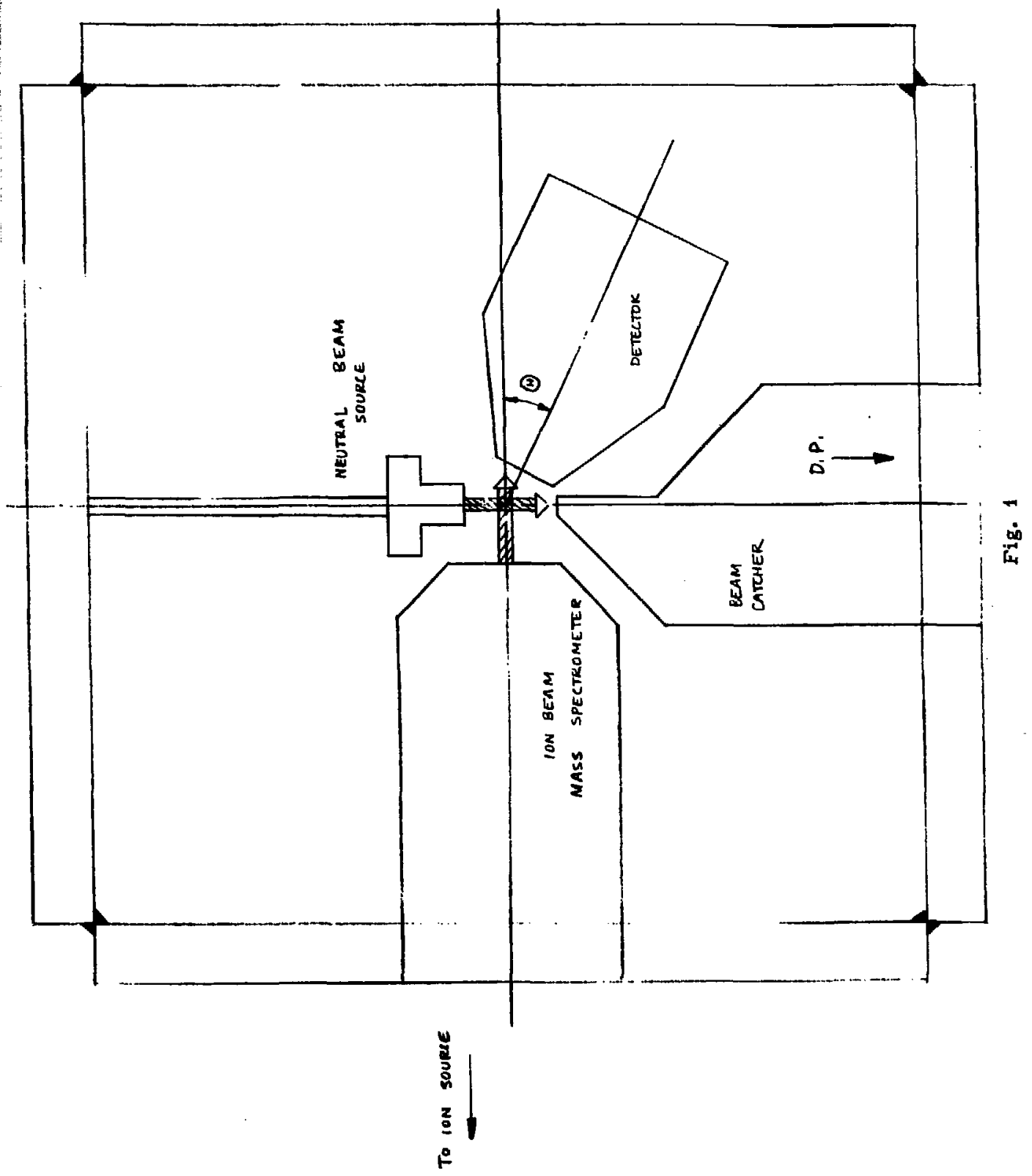




\section{Detailed Description of Components}

This chapter is devoted to a detailed discussion of the various components employed in crossed beam studies of ion: molecule reactions with this apparatus. Since the morphology of this apparatus is quite similar to that described in Gentry's report, we refer the reader to his work for a detailed description of the vacuum chamber and pumping system.

\section{A. Main Chamber and Detection Chamber}

The main chamber for this apparatus is essentially identical to that in the Gentry apparatus and, in fact, predates that chamber. A significant difference in the present apparatus concerns the fact that continuous inside welding of the main chamber was not performed although such fabrication is considered desirable for high vacuum work. We have not, however, suffered from unduly long pumpdown times and pressures as low as $4 \times 10^{-7}$ torr can be achieved routinely in the main chamber.

A significant modification to the present apparatus concerns the manner in which the product detection system is mounted. In order to assure a high degree of cleanliness in the product detection system, a valvable, differentially pumped detection chamber was constructed. The manner in which this was accomplished is shown clearly in Figure 2 which portrays the assembly of the reaction chamber. The 
Figure 2. Reaction chamber assembly drawing.

1. Reaction Chamber

2. Rotating Detector Lid

3. Veeco MI-75 Ion Pump

4. Chamber Support P1ate

5. Graduated Inner Ring

6. Bearing Retainer Ring

7. Sprocket

8. Kaydon Real-S1im Ball Bearing

9. Tec-Ring \#A01310, Fluorocarbon Corp.

10. Boston roller chain \#50,5/8 pitch

11. Chamber window flange

12. Chamber window

13. QPMS Chamber

14. Detection Chamber

15. Flanged Tee

16. Drive Sprocket Adapter

17. Drive Sprocket

18. Camrol Cam Follower

19. Blind Flange

20. Gate Valve

21. Front Plate

22. LBL Wilson seal 


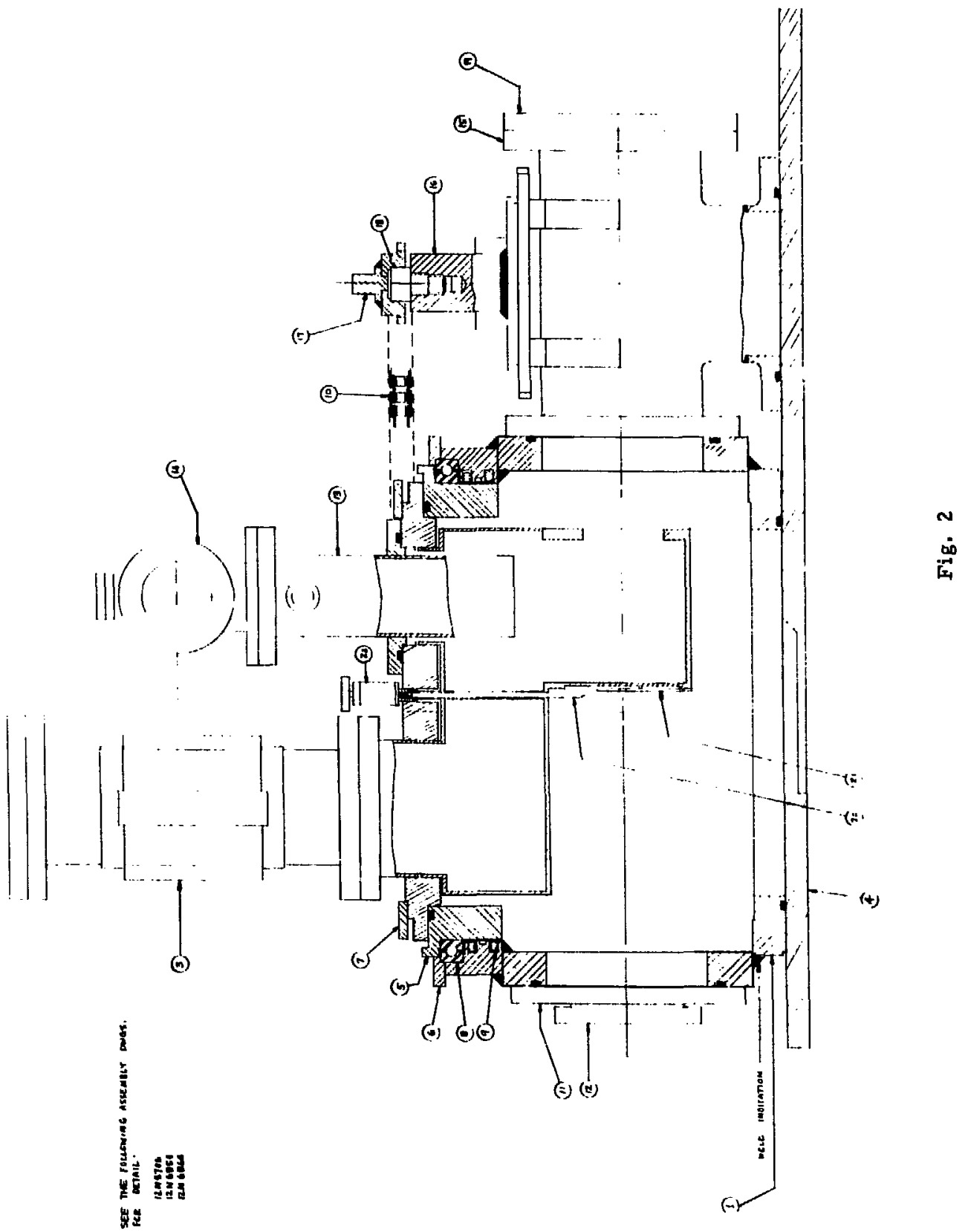


detection chamber is fabricated from type 304 stainless steel and inside Heliarc vacuum-tight welding was employed wherever feasible to eliminate virtual leaks. The chamber was welded to the top plate of the rotating 1 id via 1/8" wall stainless steel tubes; this technique results in application of less stress during the final assembly of the chamber to the lid. Because the final machining is done after welding, the amount of stress in the unit must be quite sma11, otherwise the act of machining results in uncontrolled stress relief and unpredictable changes in component alignment.

The detection chamber includes a sliding gate valve which can be actuated from outside the vacuum chamber. In order to ensure smooth sliding action and a good vacuum seal, it was necessary to apply a thin coat of Apiezon-L grease to the Viton-A 0-ring; attempts to omit the grease were unsuccessful because the rolling friction which resulted was great enough to tear large pieces from the o-ring. The face of the sliding valve which contacts the 0 -ring is polished to a mirror finish and the other side of the sliding surface which is retained by another stainless steel plate is covered with Teflon to allow for smooth action and to prevent spontaneous vacuum welding of the stainless steel pieces. The Teflon coating is removed from the immediate area of the detector aperture to prevent small pieces of the insulator from accumulating in the detector aperture. The entire front plate of the detector chamber is covered with a copper plate in which a non-inductively wound cartridge heater is encased. This heater remains on permanently to prevent insulating deposits from adsorbing on the front aperture surface. 
The determination of the location of the detector aperture in the chamber requires great care to insure that its vertical position is correct and that the 1 id rotates about the collision volume quite accurately. The vertical position of the hole was determined at assembly; the nominal location of the hole based on measurements of the thrust bearing and associated mounting components was determined and a reference 1 ine was scribed on the front of the detector chamber prior to machining that face. After all machining had been completed except for the boring of this aperture, the chamber was installed in the apparatus and pumped down to allow the bearing, the Tec-rings, and associated hardware to seat with respect to one another. Then, using the scribe marks on the opposite faces of the main vacuum chamber, a cathetometer was aligned with respect to these points of reference. The vertical location of the hole could then be determined within .005" in this manner.

The procedure required to find the center of rotation of the lid was slightly more complicated. Using the same cathetometer alignment as before, a thread was suspended from the top plate of the rotating lid. Its location with respect to the cathetometer cross hairs at a particular laboratory angle was noted and the lid was then rotated by $180^{\circ}$. If the cathetometer was aligned with respect to the center of rotation of the lid, that is, with respect to the reference scribe marks on the main vacuum chamber, then one would expect the thread to be displaced an equal amount with respect to the cathetometer cross hairs but in the opposite direction. By choosing appropriate pairs of points and using the fact that the lid could 
be rotated through $360^{\circ}$, the center of rotation was found quite easily. If a mechanical component had been present to prevent rotation of the lid through $360^{\circ}$, a second cathetometer, sighted in at $90^{\circ}$ with respect to the first would be required, but the same general method would be applicable. This method of determining the center of rotation indicated that it was coaxial with the reference axes of the main chamber and associated components within .005".

The accurate alignment of the energy analyzer and yuadrupole mass filter with respect to the collision center and with respect to one another was facilitated by careful machining of the detector chamber. The analyzer mates to the detector chamber through a precision bore machined concentrically with respect to the detector aperture. The distance from the back surface of the detector chamber to the vertical axis which references the mass filter and scintillation detector was determined at assembly with a special fixture and all dimensions on the energy analyzer were determined with respect to this value. To ensure reproducible placement of the analyzer, a 1/8" dowel pin is provided in the detector chamber and a corresponding $1 / 8$ " slot in the analyzer mounting plate creates a precise mechanism for positioning the components.

A final point concerns the vacuum system for the detector. The only aperture separating the detector chamber and the main chamber is the $1 / 8$ " aperture. The conductance of this orifice is about $31 \mathrm{iter} / \mathrm{sec}$ so that a one order of magnitude pressurc differential can be achieved with a rather low speed pump. We have chosen a 75 liter/sec ion pump manufactured by Vecco which 
pumps rare gas atoms efficiently. The ultibate pressurc which we can obtain in our present chamber is $3 \times 10^{-8}$ torr with no detector components in the chamber and $1 \times 10^{-7}$ torr with the components installed. This pressure is not optimal because the fabrication of detector components did not consider the best material selection for ulkra-high vacuum systems. llowever, these pressures are certainly low enough to reduce the background ion-molecule reactions to acceptable jevels.

\section{B. Ion Bean Sources and Focusing System}

\section{Electron Impact Source}

In a low energy ion beam apparatus, the ion source is of particular importance because large ion currents are required and small energy spread of the beam is also desirable to provide well-defined initial kinetic energy conditions. Although the original device was conceived with an energy selector in the beam preparation and transport system, we have not implamented this feacure and our momentum analyzer was designed to operate at high energy for good mass discrimination. Corsequently, significant effort was expended in developing an ion source which was mechanically simple, reliable and which provided high intensity, space charge limited ion beams with narrow energy spread. The design we chose is an elaboration of an electron impact source used by Udseth, Gentry, and Giese ${ }^{l}$ for production of low energy proton beams. The source is shown in Figure 3 and its construction and operation are described below. The ion production region of the source is effectively the anode of an electron gun formed by placing a 
Figure 3. Electron Impact Ion Source

1. Radiation Shield

2. Tungsten Cathode

3. Feedthru Flange

4. Stupakoff glass to metal seal

5. Gas Inlet

$12 N 7073$ Filament Chamber

12N7082 Insulating Flange

12N7092 Source Body

12N7101 Inner Conductor

12N7121 Outer Conductor

12N7131 Cathode Mounting Plate

12N7141 Cathode Support

12N7151 Anode Needle

12N7161 Anode Tip 

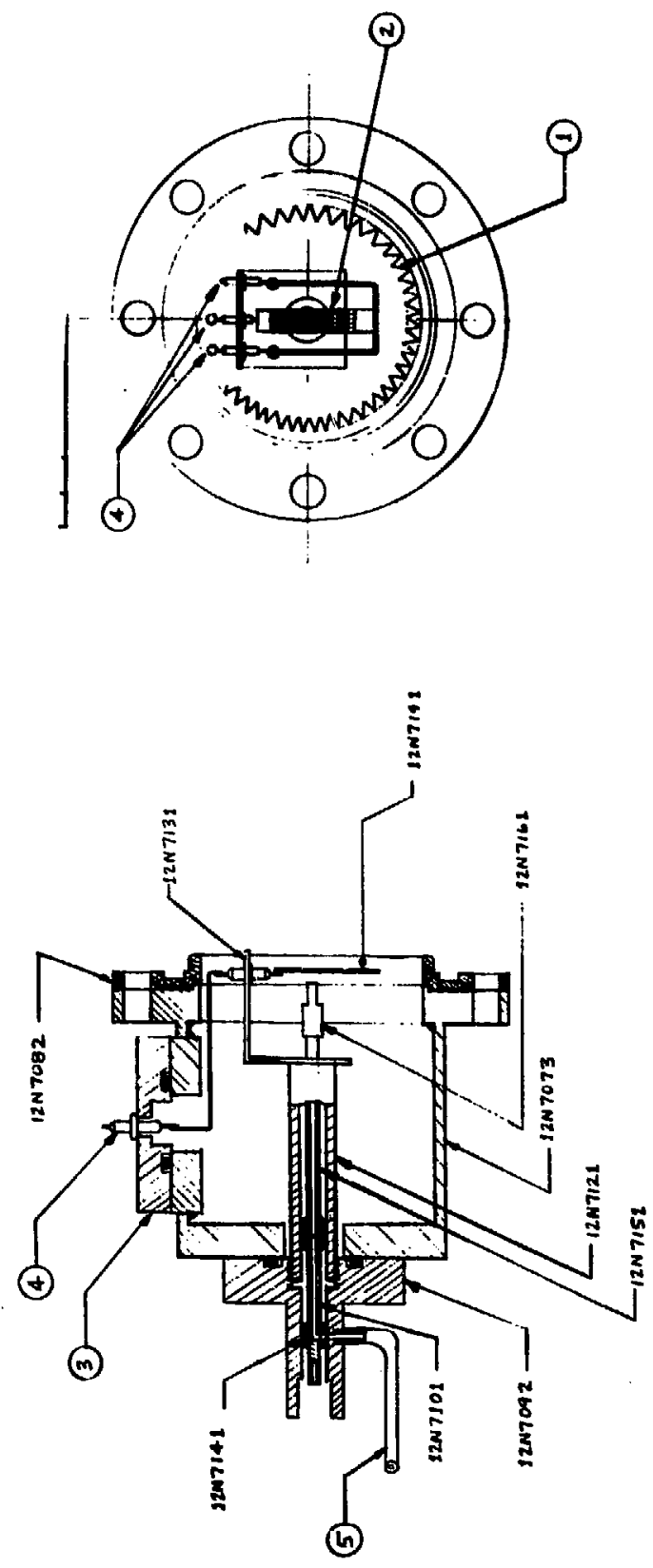

$m$ 
strip of high transmission tungsten mesh (Buckbee-Mears (:o.) in front of a molybdenum-tipped stainless steel tube with inside diameter $1.5 \mathrm{~mm}$. The anode tip is shown in Figure 3 as part 12N7161 and the cathode support and cathode strip are pare $12 N 7141$ and part 2 respectively. The gas to be ionized enters the source through the inlet tube $\underline{5}$; a potential equal to the desired ion energy is appliud to the anode and the cathode is biased negatively with respect to the anode. The cathode is heated with a DC power supply capable of providing up to 10 amps and electrons are emitted from the cathode by thermionic emission. The electrons are accelerated toward the anode where ionization takes place primarily within the equipotential volume of the anode tube. The ions are extracted by the same potential which accelerates the electrons and the ion beam focusing system transports the beam to the momentum analyzer.

The cathode grid consists of a strip of 301 line/inch, $.003 "$ linewidth and .001" thickness tungsten mesh fabricated by photochemical etching. A piece of dimension $5 \mathrm{~mm} \times 25 \mathrm{~mm}$ is spotwelded to small tantalum strips which are in turn spotwelded to the cathode support and glass to metal Stupakoff seals. Earlier designs incorporated the addition of a tantalum spring to keep the cathode taut during operation; this feature was included since it was felt that the degree of uniformity of the extracting electric field influenced the energy spread of the ions. However, energy analysis of the ion beams produced with this source and with a source with no spring did not reveal significant improvements so the spring has been omitted in later versions. 
This particular source design was chosen because of the possibility of very low energy spread ion beams resulting from ions formed in the equipotential anode region. This criterion is fulfilled only for ions which are produced with no kinetic energy release, i.e., parent molecular ions or rarc gas aromic ions. Kinetic energy release imparted to atomic ions produced by dissociative ionization of parent molecules results in kinetic energy spreads much greater than intrinsically provided by the ion source. The intrinsic spread of the source has heen determined by performing energy analysis on beams of $\mathrm{N}_{2}{ }^{+}$ and $\mathrm{H}_{2}{ }^{+}$; in these cases, full width half maximum (linim) energy spreads as low as $0.25 \mathrm{eV}$ have been determined. This performance is considered quite satisfactory and suggests that the combination of electric fields present in the ion production region yields a yery homogeneous potential distribution.

Filament life with this ion source is quite long in general, with up to 160 hours of productive beam generation time recorded. Occasionally, ion impact on the cathode results in burning a hole through the mesh and in cases where ions are produced by dissociative ionization of a parent molecule, a time-dependent shift in the nominal energy of the ion beam occurs. This effect presumably arises from a surface interaction of the cathode and is rather undesirable; this problem can be solved in general by replacing the cathode at regular intervals.

The ion source appears to be capable of producing space= charge limited beams of molecular ions and we have generated beams of $\mathrm{N}_{2}^{+}, \mathrm{H}_{2}^{+}$, and $\mathrm{C}_{2} \mathrm{H}_{4}^{+}$with currents in the $1 \times 10^{-9}$ a range at $1 \mathrm{eV}$ laboratory energy. Ion currents for atomic ions 
such as $\mathrm{N}^{+}$are generally one order of magiatude smaller than the parent molecular ion current. The source also has capabilities of functioning as a rudimentary chemical ionization source and intense beams of $\mathrm{H}_{3}{ }^{+}$and $\mathrm{C}_{3} \mathrm{H}_{5}{ }^{+}$have been produced in the following reactions:

$$
\begin{gathered}
\mathrm{H}_{2}^{+}+\mathrm{H}_{2}+\underline{\mathrm{H}}_{3}^{+}+\mathrm{H} \\
\mathrm{C}_{2} \mathrm{H}_{4}^{+}+\mathrm{C}_{2} \mathrm{H}_{4} \rightarrow \mathrm{C}_{4} \mathrm{H}_{8}^{+}+\mathrm{C}_{3} \mathrm{H}_{5}^{+}+\mathrm{CH}_{3} .
\end{gathered}
$$

While these reactions proceed with large cross sections, the energy spreads of the resulting ion beams are quite large and the plasma sources developed by Field ${ }^{2}$ and lutrell and co-workers ${ }^{3}$ are more suited for high quality beams of this type because the energy spreads in their sources are determined by the tempc turc at which thermal equilibrium is achieved in the plasma.

\section{Microwave Discharge Source}

This source, developed. by M. Chiang and described in his thesis ${ }^{4}$ will be discussed only briefly. Discharge sources offer particular advantages for certain ion-molecule experiments, but little work on adapting such sources for our low encrgy studies has been done. The microwave discharge source used in this laboratory is of interest primarily because it provides one with a source of ground state ions. We have used this source without modification to produce ground state $\mathrm{N}^{+}$beams for comparison with results obtained with our electron impact generated ion beams, known to contain contributions from the metastable excited states $N^{+}\left({ }^{1} D\right)$ and $N^{+}\left({ }^{1} S\right)$. The only modification required for this source is use of a conical extractor 
to facilitate removal of ions from the plasma meniscus. Several low energy experiments have been performed with the microwave discharge source and the following observations recorded:

1. The energy spread of $N^{+}$ions from the source is somewhat broader than from the electron impact source, thereby making studies at the lowest energies energies of marginal value.

2. The "plasma potential" which results from local deviations of the plasma from electrical neutrality is quite large ( $\geq 30$ volts) and the source electrodes must be floated at large negative potentials to compensate for this effect.

In general, however, despite these two difficulties, intense, stable beams can be produced with this source. A DC discharge source which seems appealing has been developed by Wahl in and Menzinger 5 and has been employed by the Cross group at Yale and the Henglein group at Berlin with moderate success. Energy spreads from this source appear to be quite narrow because the ions are extracted from the "positive. column" of the discharge, a fairly equipotential region of the discharge, but the excited state composition of beams from this source is not characterized.

\section{Ion Beam Analysis and Focusing System}

The requirements on the ion beam transport system are similar to those of the high energy machine in this laboratory. Beams of high intensity, small energy and angular spread and good mass resolution are desired. Good beam formation and 
collimation ion optics are of special importance in low energy work where space charge defocusing is jarticularly troublesome. The ion beam mass spectrometer is divided into three sections: the initial focusing stage extracts the ions from. the source and focuses the ions into a beam appropriate for momentum analysis. The momentum analysis is performed with a $60^{\circ}$ sector magnet comprising the second stage of the source mass spectrometer. The final focusing stage transports the momentum analyzed beam to the decelerator which retards the ions to their final energy with respect to the collision center which is at ground potential.

The overall design of the ion source mass spectrometer is shown in Figure 4 and 5 and Figure 6 shows an assembly of the initial focusing stage. Figure 4 should be consulted in the following discussion. Ions formed in the source are extracted and collimated by the double aperture lens $\underline{3}$. The einzel iens $\underline{4}$ provides the beam with corrective focusing prior to injection into the momentum analyzer. The parallel grids, elements 5, accelerate the beam from the nominal energy of the second aperture to the energy at which momentum analysis is performed. Up to this point in the beam transport system the bean possesses cylindrical symmetry; however, because the momentum analysis magnet focuses only in the horizontal plane, we desire to eliminate losses resulting from ions with vertical velocity components. A quadrupole doublet 6 converts the beam of square cross section as it emerges from the parallel grids to a line-focused beam as it enters 
the mass spectrometer chamber. The electromagnet (not shown in Figure 4) deflects the ions in the $60^{\circ}$-angle flight tube 7 according to their momenta. After momentum analysis, the beam is refocused to cylindrical symetry with another quadrupole doublet 9. An einzel lens 10 operated in the retarding mode focuses the beam and a set of four deflection plates 11 align the beam with the decelerator 12 which retards the ions from the momentum analysis energy (typically $300 \mathrm{~V}$ ) to ground potential. Figure 7 shows an assembly drawing of the final focusing stage. Figure 5 shows a block diagram of the power supplies for the ion source mass spectrometer indicating the interrelationships among the potentials applied to various ion optical elements.

\section{Design Features}

The initial focusing stage up to the quadrupole doublet is designed according to the specifications given by Gentry. The double aperture lens is designed according to the graphs given in Spangenberg; a ratio of 1:4 for the first and second aperture potentials collimates the beam, i.e., focuses the beam at infinity. ${ }^{6}$ In practice, ratios closer to $1: 6$ or $1: 8$ have produced more intense beams with no apparent loss of quality.

The quadrupole doublets were designed as indicated by Lu and Carr ${ }^{7}$ and implemented by Gentry. A significant departure from Gentry's geometry was chosen, however, to correct for the strong coupling between the first and second quadrupoles. The beam half-angle at crossover was selected to be $1.8^{\circ}$, 
ligure 4. Ion Source Mass Spectrometer

1. Ion Beam Source Chamber

2. Beam Extractor

3. Double Nperture Lens

4. I:inzel Lens

5. Accelerating (irids

6. Quadrupole Lens lilements

7. Momentum Nnalyzer Chamber

8. Final Focusing Beam Chamber

9. Quadrupole Lens Elements

10. Einze1 Lens

11. Deflection Plates

12. Retarder

13. Ion Beam Chamber 


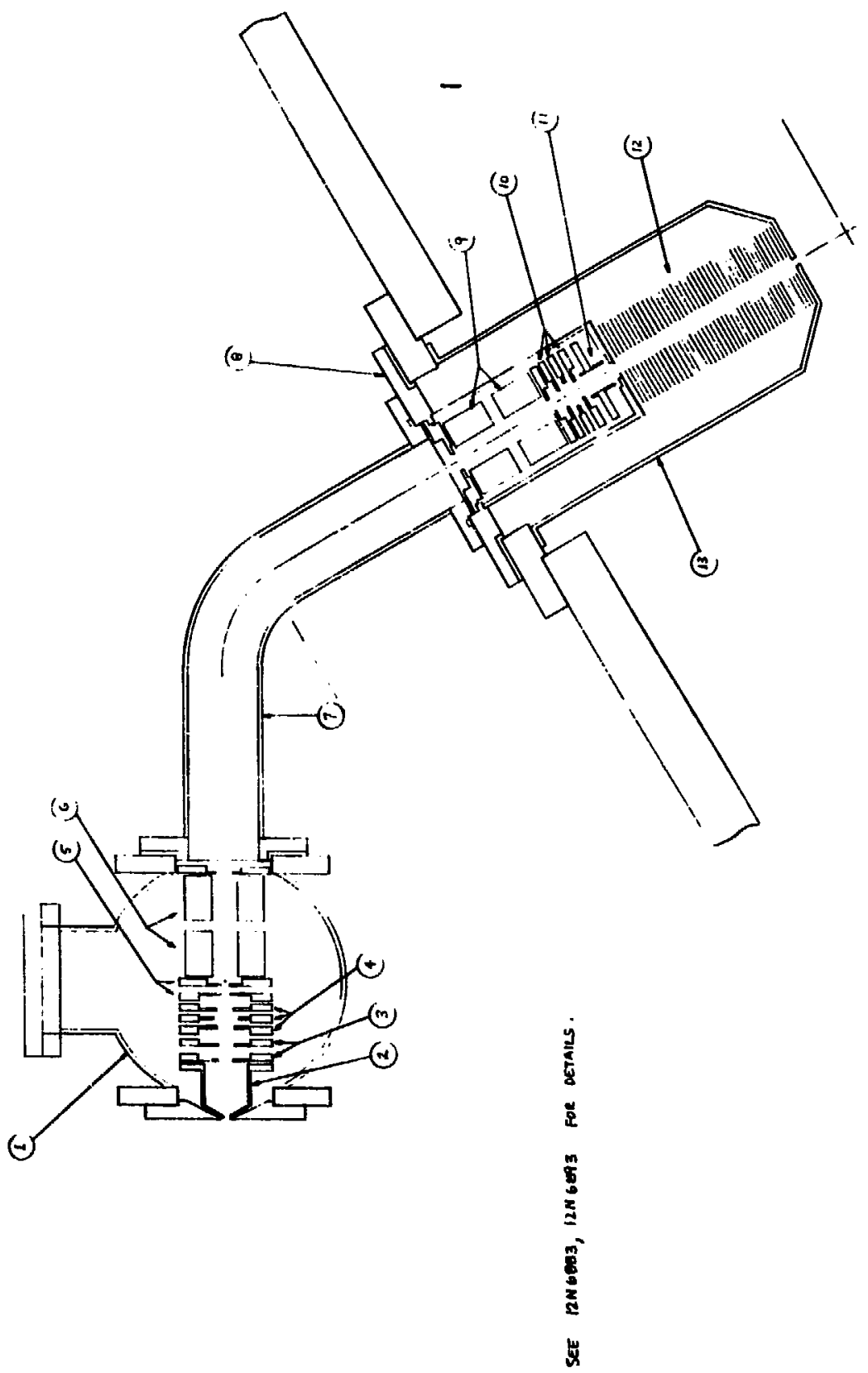

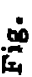


Figure 5. Block diagram showing power supply connections for the ion source mass spectrometer. The ion source is shown schematically in this figure. 


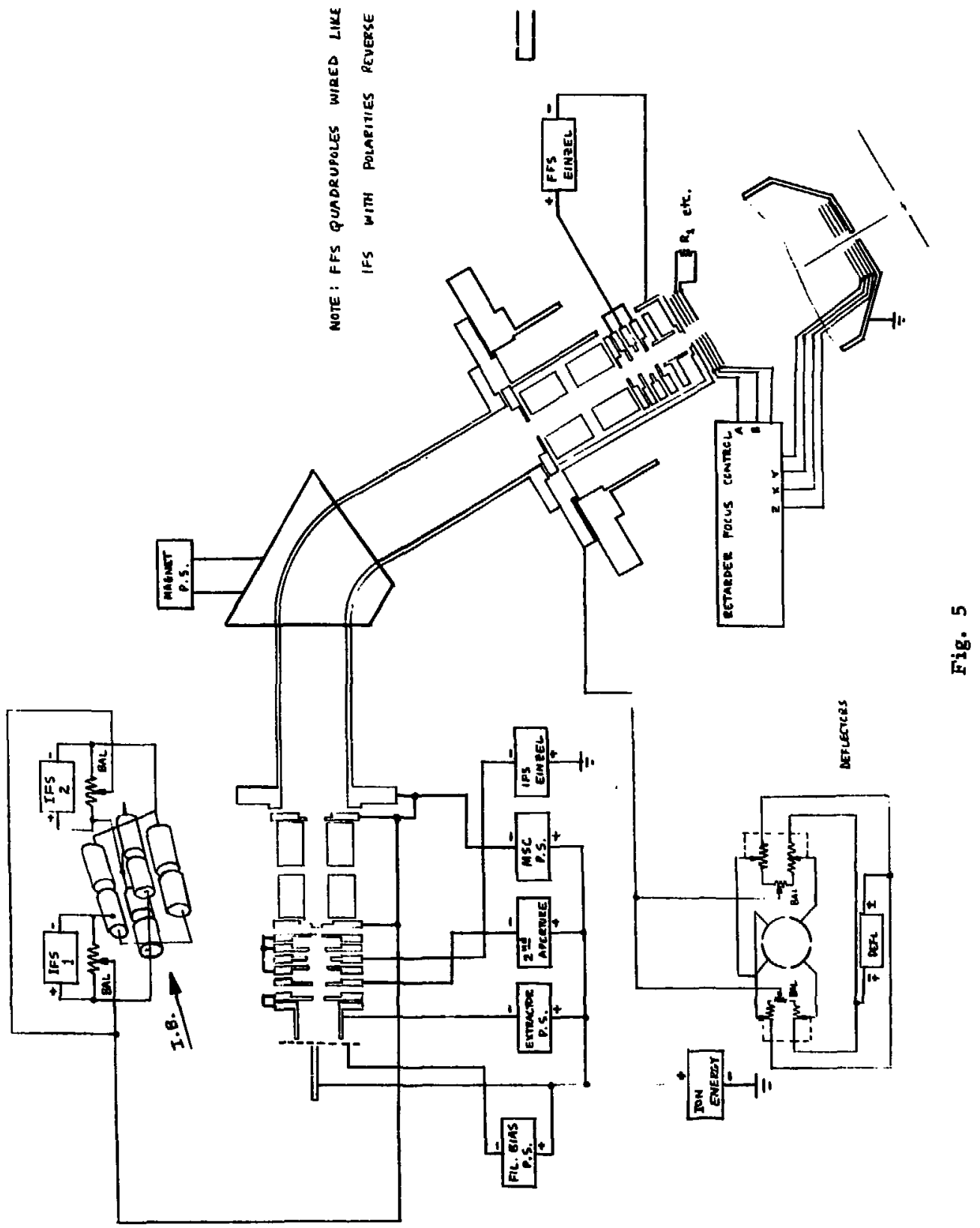


Figure 6. Assembly drawing of the initial focusing stage of the ion source mass spectrometer.

1. Extractor

2. Aperture

3. Second Aperture

4. Einzel Lens -1

5. Einzel Lens -Center

6. Einzel Lens -3

7. Accelerating Grid 1

8. Accelerating Grid 2

9. Quadrupole Rods

10. Element 10

11. Momentum Analyzer Chamber

12. Momentum Analyzer S1it 


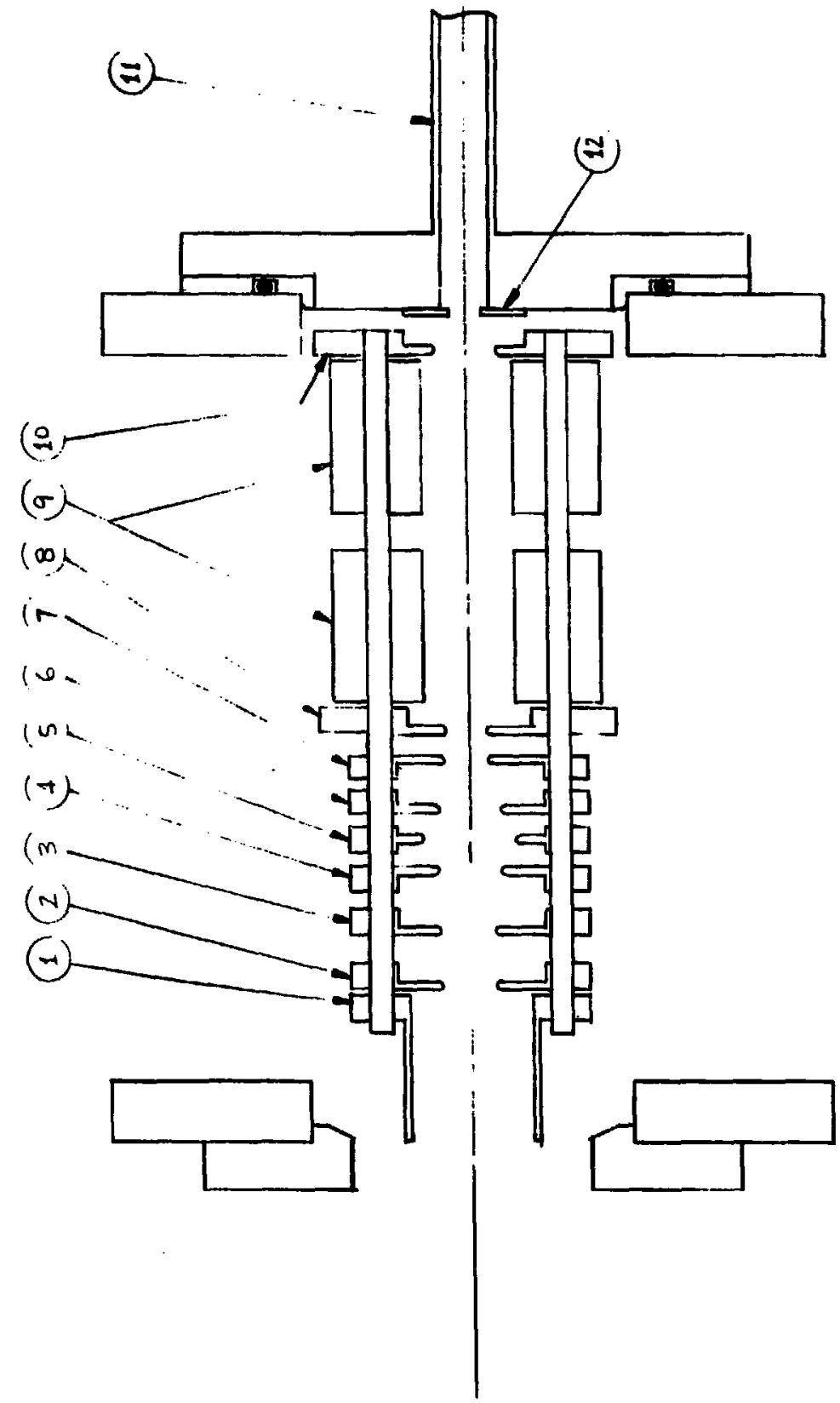

0 
Figure 7. Final focusing stage assembly drawing

1. Momentum Analyzer Chamber

2. Momentum Analyzer Exit S1it

3. Analysis Chamber

4. Focusing Element Mounting Plate

5. Quadrupole Rod

6. Element 3

7. Element 4 - Einzel Lens

8. Element 5 - Einzel Lens

9. Element 6 - Einzel Lens

10. Deflection Plate

11. Retarder Lens Element

12. Retarder Lens Retainer

13. Retarder Entrance S1it

14. Ion Beam Chamber

15. Adapter Flange 
(A)

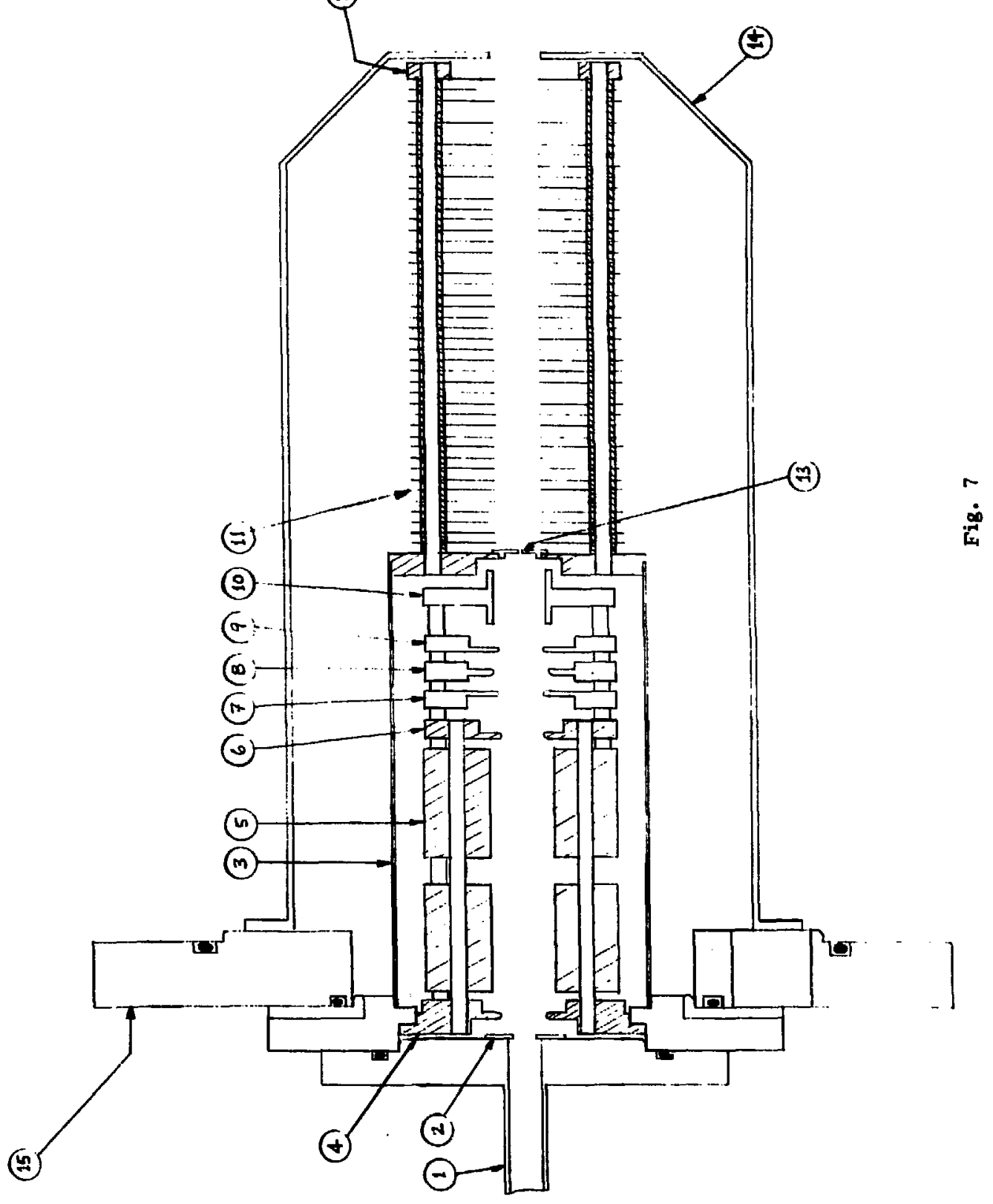


the quadrupole length $1.25 "$ and the spacing between the individual elements of the doublet 0.312". With 0.750" diameter rods, $S / D=0.25$ where $S$ is the spacing and $D$ is the quadrupole lens element length. For a beam which has dimensions .200" $x$ $.200 "$ ", the crossover point must therefore be $0.537 "$ from the exit of the lens. Using these figures we find that the applied potentials measured with respect to the momentum analysis potential $V_{M S C}$ should be $\pm .05 V_{M S C}$ for the first quadrupole and $\pm .034 V_{M S C}$ for the second quadrupole. The first quadrupje then converts the beam of square cross section to a $r$ ibbon shape; the second quadrupole reverses the potentials but to a smaller extent such that the net reshaping of the beam occurs. Because of the symmetry of the initial and final focusing stage quadrupoles and the momentum analyzer, the final stage quadrupoles must undo the focusing generated in the initial stage. We have discovered that the doublets are most efficiently operated in this symmetric mode, but applied potentials are generally $50 \%$ to twofold higher than one calculates. The initial focusing stage, shown in more detail in Figure 6 , is supported by four rods which thread into the momentum analysis chamber 11 ; the details of this construction are suppressed in Figure 6. The ion optical elements themselves are supported by precision ground alumina rods and can be positioned to within .002" quite readily. The elements are aligned on the rods using special removable spacers machined from copper; these spacers are .068" thick and the cylindrical elements in the initial focusing system are separated by the thickness 
of one spacer with the exception of the extractor and the double aperture lens elements which are separated by two spacers. The lens elements are fabricated from aluminum with the exception of the extractor, the first aperture, and the accelerating grids. The latter two elements are machined from stainless steel so that high transmission gold mesh can be spot welded across the square apertures; the first three elements in the initial focusing stage are fabricated from stainless steel to reduce erosion of the lens elements from sputtering by intense ion beams. All lens elements arc gold plated and, in addition, a thin coating of colloidal graphite (Aquadag) applied to all elements improves stability. This coating must be renewed frequently for work with low energy ions; instability of the beam current is a general indication of dirty ion optics. Dirty conditions are most frequently encountered in the initial focusing system, thereby requiring frequent cleaning of this section of the apparatus. We have found that the final focusing stage remains cleaner for much longer periods with only infrequent cleaning required.

The initial focusing stage extracts low energy ions from the source and accelerates and focuses them to approximately $300 \mathrm{~V}$ for momentum analysis by a $60^{\circ}$ sector magnet. This magnet is designed with very simple criteria: the entrance and exit boundaries to the field should be approximately perpendicular to the nominal trajectory through the field and the image and object distances should be equal. Satisfactory solutions to these criteria can be determined by considering the Newton lens equation for a focusing lens: 8,9 


$$
\begin{aligned}
& \left(\ell_{0}-g\right)\left(\ell_{I}-g\right)=f^{2} \\
& \ell_{0}=\text { object distance } \\
& \ell_{I}=\text { image distance } \\
& g=r_{0} \cot \phi \\
& f=r_{0} \csc \phi \\
& r_{0}=\text { cyclotron radius } \\
& \phi=\text { magnet "wedge" angle }
\end{aligned}
$$

For our geometry we have chosen $\phi=60^{\circ}, r_{0}=8 \mathrm{~cm}$, the nominal radius of curvature an ion required to pass through the analyzer, and $\ell_{0}=\ell_{I}$. We find, then, that the image and object distances equal $\sqrt{3} \mathrm{r}_{0}$ or $13.86 \mathrm{~cm}$. We can calculate the dispersion of this magnetic lens by computing the displacement $d_{I}$ which results from a displacement $d_{0}$ of the object from the nominal trajectory which focuses an ion of mass $m$ and velocity $v$ :

$$
d_{I}=2 r_{0}\left(\frac{\delta m}{m}+\frac{\delta v}{v}\right)-d_{0} .
$$

The terms in $\delta m$ and $\delta v$ are symmetric and reflect the momentum analysis capabilities of the system. Now $\delta v / v=\delta E / 2 E$ and $\delta E$ is the intrinsic energy spread of the ion source, assuming that the accelerating field for the momentum analyzer is uniform. Since the momentum analyzer operates at $E \approx 300 \mathrm{v}$, the $\delta \mathrm{v} / \mathrm{v}$ term is small compared to $\delta \mathrm{m} / \mathrm{m}$ and consequently the high energy operation of the analyzer is that of a mass spectrometer. In fact, we can write

$$
\frac{m}{\Delta m}=\frac{2 r_{0}}{d_{I}+d_{0}}
$$


If $d_{I}$ and $d_{0}$ represent the image and object slit widths, then $d_{I}=.050 "$ and $\dot{d}_{0}=.080^{\prime \prime}$ results in $\mathrm{m} / \Delta \mathrm{m}=48$.

The magnet is machined from mild steel and is nickel

plated to resist corrosion. The magnet coils consist of 1000 turns of $16 \mathrm{ga}$. copper wire wound in two pancake coils near the gaps and potted in epoxy resin. The magnet current required to focus singly charged ions of mass $M(a m u)$ at analysis energy $E_{A}(\mathrm{eV})$ has been found to obey the following relation:

$$
i_{\text {riagnet }}(\text { amp })=\frac{\sqrt{M_{A}}}{57} \text {. }
$$

The $60^{\circ}$ flight tube itself is electrically isolated from other elements in the ion optical train by using nylon spacers and Mylar shims between the tube and the magnet pole faces. This isolation allows the momentum analysis to be performed at energies different from the ion source potential and allows the collision center to be at ground potential as well.

Ions which have been momentum analyzed at $\sim 300 \mathrm{eV}$ exit the analyzer in a ribbon-shaped beam which is reconverted to approximate cylindrical symmetry by another quadrupole doublet, element $\underline{9}$ in Figure 4 , focused by an einzel lens 10 and deflected onto the entrance aperture of a decelerator. The deflection plates 11 are fabricated by quartering a cylinder and biasing the plates about the momentum analyzer potential.

Perhaps the most critical element in the ion beam mass spectrometer system is the decelerator which must retard ions from the $300 \mathrm{~V}$ at which momentum analysis is performed to their nominal energy with respect to ground potential. The design 
we chose is the multi-element exponential retarder described by Vestal et $\underline{a 1}^{3}$. 10,11

In this device, the ions experience a potential distribution which decelerates them in the following manner:

$$
V=-v_{0} \exp (-\alpha z)
$$

where $z$ measures motion along the beam direction. Such a simple analytic form allows one to solve the paraxial ray equation of ion optics analytically for $r(z)$, the radial displacement from the retarder center-line as a function of $z$. The paraxial ray equation is given by 12

$$
r^{\prime \prime}+\left(V^{\prime} / 2 V\right) r^{\prime}+\left(V^{\prime \prime} / 4 V\right) r=0
$$

where the primes denote differentiacion with respect to $z$. The solution $r(z)$ can be written

$$
r(z)=C \exp (\alpha z / 4) \sin (\sqrt{3} \alpha z / 4+\phi) .
$$

The following symbols require definition:

$$
\begin{aligned}
& c=(2 / \alpha)\left[\left(4 \theta_{0}{ }^{2}-2 \theta_{0} r_{0} \alpha+r_{0}{ }^{2} \alpha^{2}\right) / 3\right]^{1 / 2} \\
& \phi=\sin ^{-1}\left(r_{0} / C\right) \\
& r_{0}=\text { initial displacement of entering ion } \\
& \theta_{0}=\text { initial injection angle. }
\end{aligned}
$$

The first focus can be obtained by setting the argument of the sine function equal to zero. $\phi$ can also be set equal to zero by invoking the initial condition that injection be on the retarder axis. Then $z_{\Gamma}=4 \pi / \alpha \sqrt{3}$ and at focus 


$$
V=-V_{0} \exp [-4 \pi / \sqrt{3}] \approx-.0007 V_{0}
$$

Thus for $300 \mathrm{eV}$ analysis, $V\left(z_{f}\right) \approx-0.2 \mathrm{eV}$. The angular and radial magnifications of the retarder can be computed readily by examining the solution to the paraxial ray equation

$$
\begin{aligned}
& M_{\theta}=\left(\theta_{f} / \theta_{0}\right)_{r_{0}=0}=e^{-\pi / \sqrt{3}} \approx-6.1 \\
& M_{r}=\left(r_{f} / r_{0}\right)_{\theta_{0}=0}=e^{-\pi / \sqrt{3}} \approx-6.1
\end{aligned}
$$

The magnifications are equal and the image is inverted and magnified. Thus a beam of dimension $3 \mathrm{~mm}$ and FWHM angular divergence of $3^{\circ}$ at the collision center requires the object to be collimated to $0.5^{\circ}$ and $3 \mathrm{~mm}$. In general, we have found that these criteria are fulfilled by the virtual aperture arrangement of the final focusing stage quadrupole doublet and einzel lens. The einzel lens is operated in a retarding mode (center element at a positive potential with respect to the momentum analyzer potential) to act as a short focal length lens.

The Abbé sine law allows us to determine the minimum attainable magnifications for a given amount of retardation:

$$
r_{0} \theta_{0} F_{0}=r_{f} \theta_{f} \sqrt{V_{f}} .
$$

We therefore find that

$$
M_{\theta}^{2} M_{r}^{2}=v_{0} / v_{f} .
$$


Consequently, a decrease in image magnification can only be obtained at the cost of increased angular magnification and vice versa. This retarder constrains the magnifications to be equal and this property is quite desirable for our experiments.

The retarder design was taken directly from the work of Vestal et al. with on $1 y$ minor modifications. ${ }^{3}$ Large apertures were chosen to ensure the validity of the paraxial approximation; the aperture size was chosen to be $0.500 "$ and the retarder plates were fabricated from .010" thick type 302 stainless steel. The number of plates chosen was 43 , spaced by $0.125^{\prime \prime}$. A focus at the collision center was required at a distance of $5.75^{\prime \prime}$ from the entrance plane to the retarder. Thus, the exponential parameter $\alpha$ was chosen to be $1.262 \mathrm{in}^{-1}$; appropriate $1 \frac{8}{6}$ metal film resistors were chosen for an integral voltage divider to supply the necessary exponential potential drop along the retarder axis. The first two and last three retarder elements are connected to potentiometers mounted externally to the vacuum envelope to provide empirical correctiors to the focusing created by fringing fields in the $\mathrm{re}^{+}$rder.

The performance of the retarder has been quite satisfactory; essentially complete transmission of low energy ion beams has been observed with no degradation in performance over six months of continuous operation. The unit is cleaned as a single piece when necessary; mounting the unit on alumina rods and precision ground alumina spacers eliminates 
any significant outgassing problem and the insulators are well shielded from the beam, thereby eliminating problems with surface charge buildup.

Operation

The power supply interconnections for the ion source and ion beam mass spectrometer are indicated schematically in Figure 5. All power supplies are connected within a central control panel as in the scattering cell apparatus. A11 power supplies except the einzel lens supplies are floated at the ion source anode voltage; because the collision center is at ground potential, the nominal ion energy is determined by this anode voltage. The initial focusing stage einzel lens power supply is referenced with respect to ground and provides a negative voltage with respect to ground; the final focusing stage supply, however, provides a positive voltage with respect to the mass spectrometer analysis potential. With the exception of the einzel lens potentials, then, varying the ion source anode voltage does not change the relative focusing voltages nor affect the momentum analyzer focusing properties.

The first element of the ion source mass spectrometer is a cylindrical element which is generally held at a negative potential with respect to the ion source cathode. Voltages near $300 \mathrm{~V}$ are used typically on this element; a second aperture potential of 40-50 $\mathrm{V}$ generally collimates the beam to maximize transmission through the focusing system. The momentum analysis potential is typically 300-400 $\mathrm{V}$; these high 
voltages are found to be optimal in producing high quality beams with the exponential retarder. Under these operating conditions, momentum analysis becomes mass analysis and the analyzer does not function to reduce the energy spread of the ion source.

Quadrupole and einzel lens voltages are chosen to maximize transmission through the focusing stage. Balance helipots (see Figure 5) in the ion beam focusing control bias the quadrupoles about the momentum analysis potential and symmetric bias voltages have always been used.

\section{Ion Beam Monitors}

The total ion beam current at the collision center can be measured with a Faraday cup mounted on the sliding valve for the detector aperture. The output from this cup is fed to a Keithley $610 \mathrm{~B}$ electrometer. Because the cup is used only for diagnostic purposes in tuning the beam, no pains were taken to add retarding grids or to optimize design for $100 \%$ collection efficiency. The cup may be moved out of the beam by lifting the detector gate valve.

\section{Molecular Beam Source}

As we have indicated earlier, a crossed beam neutral source offers significant advantages over the scattering ce11, primarily associated with improved energy resolution at low energies arising from a narrower distribution of initial relative velocity vectors. However, one loses significant intensity when a crossed beam is employed because the path 
length over which the ion beam is attenuated by the neutral beam is an order of magnitude smaller than in the collision ce11. In addition, much higher gas densities may be used in a collision cell, thereby increasing the scattered intensity compared to the undeflected intensity. We have chosen to fabricate a rudimentary supersonic nozzle to overcome the intensity problems of an effusive beam as well as providing us with a more nearly monoenergetic source of neutral species.

Whereas an effusive source operates under molecular flow conditions in which the mean free path of the neutral species must be greater than or equal to the orifice dimension, a supersonic nozzle operates in the hydrodynamic flow regime where mean free paths are much smaller than orifice dimensions. This technique was suggested 25 years ago by Kantrowitz and Grey ${ }^{13}$ as a means of producing intense beams of atoms and Kistiakowsky and slichter ${ }^{14}$ attempted to use the method, but the significant experimental hurdles associated with the required high pumping speeds were not adequately solved until 10 years ago. Noteworthy progress in the production of nozzle beams is still being made. Although the production of high quality, high intensity nozzle beams remains more an art than a science, significant advances in beam dynamics have been achieved along two distinct pathways. These two schools of thought, associated with the names of Campargue 15 and Bosse 16 respectively, differ in the engineering associated with beam production but the physics of beam formation is the same and we refer the reader to the literature for differences between the two schools. 
Supersonic nozzle beams are formed by expanding a gas at a high pressure, say $\$ 100$ torr, into a vacuum chamber under conditions of constant entropy. The constancy of the entropy of the gas is characteristic of an adiabatic expansion and the thermodynamics of adiabatic expansions can be applied profitably to the process. Roughly speaking, the temperature of the gas decreases because of the increase in volume upon expanding; this temperature refers to the "transverse temperature" of random motion transverse to the direction of hydrodynamic flow streamlines. A consideration of a flow streamline indicates that the enthalpy of the gas is converted into translational motion; ${ }^{17}$ the enthalpy associated with random thermal motion can increase the mean speed of a particle with respect to the $\sqrt{2 \mathrm{kT} / \mathrm{m}}$ term arising from the effusive behavior of particles under molecular flow conditions. Additionally, under the high collision frequency conditions of a nozzle bean, internal degrees of freedom may contribute to the terminal velocity of the particle. We can express the limiting behavior of the contribution of particles with structure to the terminal velocity through the relation

$$
v_{\max }=\sqrt{\frac{\gamma}{\gamma-1}\left(\frac{2 \mathrm{kT}}{m}\right)}
$$

where $\gamma=c_{p} / c_{v}$. For monatomic gases, $\gamma=5 / 2$ and $v_{\max }=$ $\sqrt{5 k T / m}$ whereas for diatomic molecules for which two rotational degrees of freedom can relax (but generally not vibrational degrees of freedom), $\gamma=7 / 2$ and $v_{\max }=\sqrt{7 \mathrm{kT} / \mathrm{m}}$. Thus, an enhancement of the maximum velocity of the particles occurs. 
We also find that the velocity distribution narrows since it depends on the local transverse temperature which in turn can be expressed in terms of a Mach number, a measure of the local speed of sound. An equivalent expression is the FWHM of the velocity distribution.

The Mach number $M$ is conventionally used as a measure of the velocity distribution from a supersonic nozzle and experimental studies have provided an empirical relationship between $M$ and the Knudsen number associated with viscous flow of the gas through the orifice. The Knudsen number, $\mathrm{K} \mathrm{n}_{0}$, is the ratio of the viscosity based mean free path of the gas to the nozzle diameter and the Mach number has been shown to obey the relation

$$
M=1.17 \mathrm{Kn}_{0}^{-0.4}
$$

at least for a monatomic gas. ${ }^{18}$ The Knudsen number exponent is genera11y given by $(1-\gamma) / \gamma$.

A more useful quantity for discussing properties of supersonic expansions is the speed ratio $w$ defined by

$$
w=m u^{2} / 2 k T
$$

where $u$ is the streamline velocity and $T$ is the local transverse temperature. In fact, the speed ratio can be shown to be related to $M$ as follows:

$$
w=(\gamma / 2)^{1 / 2} M
$$

It should be noted that Mach numbers exceeding 100 have been 
achieved in nozzle beams operating efficient1y with very low transverse temperatures.

One further advantage of supersonic nozzle beams is derived from a feature of the hydrodynamic flow which characterizes such beams. Because a beam particle experiences many collisions during the expansion process, particles of different masses tend to gain the same terminal speed which is governed by the average mass number of the gas mixture. For example, in a mixture of $18 \mathrm{Xe}$ in $\mathrm{H}_{2}$, the average mass number of the gas mixture is about 3.3 so that a Xe atom in such a mixture gains a factor of $\sim 40$ in kinetic energy with respect to the energy of a Xe atom emanating from the expansion of a pure gas. This technique of seeded beams has been used to produce hyperthermal beams of heavy atoms for collision experiments with excellent results. ${ }^{19,20}$ Another facet of beam production with mixed gases concerns the enhanced centerline concentration of the heavier species in the mixture. In principle, the centerline concentration is governed by diffusion of the lighter species in directions transverse to the centerline, so that the mass ratio of heavy species to light species should determine the enhancement. This enhancement has been observed and in fact provides a means of isotope separation, ${ }^{21}$ but its chief interest to beam experimenters concerns the possibility of high energy, high intensity beams of neutral species.

The above discussion is quite abbreviated but should suggest that the use of nozzle beams is attractive for a variety of experimental techniques although engineering 
difficulties may be encountered for which definitive solutions are not yet available.

Because our apparatus lacked the capability of adding a conventional, doubly-differentially pumped supersonic nozzle source, we chose to do something which is significantly simpler, but provides us with a rudimentary approach to supersonic nozzles.

Because of the highly directional nature of supersonic nozzles, under favorable conditions one stage of differential pumping can be eliminated and replaced by a conically shaped "beam catcher" located after the beam passes through the collision region. A diffusion pump then removes the bulk of the beam material. The schematic manner in which the source and catcher are mounted is shown in Figure 1, as we indicated in Chapter I. A significant disadvantage in this method of beam production occurs because the absence of differential pumping in the source region precludes the possibility of clean modulation of the neutral beam by a mechanical chopper. In a crossed beam experiment, modulation of the neutral beam is particularly advantageous to correct for chemical reaction products generated by background molecules reacting along the entire path length of the ion beam. Because the attenuation of the ion beam by the neutral beam is low and the collision volume in which chemical reactions take place is small compared to the ion beam path length through the collision chamber, this background correction can be (and usually is) very important. We have devised a method for 
making this correction using the experimental arrangement described here and this technique will be described in the next chapter, but one should note that a mechanical modulation system coupled with gated scalers provides the best solution to the background correction problem and is planned for the near future with this apparatus.

The nozzle orifice plate itself is fabricated from stainless steel and the nozzle hole is $.025 \mathrm{~mm}$ in diameter; this small hole is machined by laser drilling at Lawrence Livermore Laboratory and the plate thickness at the point where the hole is drilled through is controlled using special machining techniques. The back of the orifice plate is relieved by grinding with a radiused wheel such that the wall thickness is .002" to .003" at the point where the hole is drilled but increases rapidly enough away from the hole to maintain structural integrity. Careful control of wall thickness at the hole is important in clean beam generation because the channel length plays a role in cluster formation and viscosity losses during expansion. Previous experience has dictated that wall thickness should not exceed two to three times the nozzle diameter so that for very small holes, necessary to reduce the gas load to the pumping system, very careful machining is required. 


\section{Product Detection System}

\section{Spherical Electrostatic Energy Analyzer}

In order to measure both energy and angular distributions of scattered ions, we have included an energy analyzer in conjunction with our rotatable mass spectrometric detector. Since we are detecting ions, we can take advantage of a large array of analyzers developed for this purpose. Retarding field energy analyzers ${ }^{22}$ are quite simple to construct and operate, but the data which one obtains from such an analyzer are integral data and must be differentiated numerically (or sometimes instrumentally) to obtain the desired flux distribution function. This procedure is fraught with difficulties and artifacts as Futrell et a1. ${ }^{3,11}$ have indicated and additionally, weak features in the energy spectrum are difficult to extract reliably in the presence of dominating intensities, e.g., backward scattering in the presence of an intense forward scattered spectator stripping peak. Deflection analyzers overcome these difficulties by producing the differential spectrum directly, but with the disadvantage of greater mechanical and operating complexity and somewhat reduced sensitivity. A number of different designs for deflection analyzers have been advanced, among the most notable the $127^{\circ}$ cylindrical analyzer of Marmet and Kerwin. 23 The cylindrical analyzer has been used quite extensively but it only provides focusing in one firection; the absence of focusing in the transverse direction results in a significant 
intensity loss. The toroidal condenser provides two dimensional focusing to overcome this loss and the degenerate case of a spherical condenser has been employed extensively as an effective deflection energy analyzer. As in the older scattering cell device in this laboratory, we have chosen to employ a spherical electrostatic analyzer as originally suggested by Purcel1 24 and extensively developed at the National Bureau of Standards by Simpson, Kuyatt and co= workers. 25,26

The trajectory of a charged particle in an electric field depends only upon its kinetic energy and net charge; consequently, one can solve the equation of motion for a. charged particle in an electric field to compute trajectories and therefore focal properties. One should note that the Newton lens equation, a form of which we wrote down for our $60^{\circ}$ momentum analyzer, also applies to this focusing device;

a complete discussion of the focusing characteristics of electrostatic analyzers is found in reference 27 . General geometric characteristics of deflecting fields derived from Gauss' law determine the radial dependence of the electric field between two concentric charged spheres and integration of the field across the gap of the analyzer allows one to determine the constant of proportionality between the energy of the ions passed by the analyzer and the applied potential. We have chosen a $90^{\circ}$ spherical analyzer for which the foci are located one mean radius $r_{e}$ from the entrance and exit boundaries to the field; under these conditions, in the absence 
of external fields which modify the boundary conditions, we can write the following relation between the applied potential and the nominal energy of transmitted ions:

$$
V_{E A}=2 r_{e}\left(\frac{1}{r_{1}}-\frac{1}{r_{2}}\right) E
$$

with $r_{e}=\left(r_{1}+r_{2}\right) / 2, r_{1}$ and $r_{2}$ being the radii of the concentric spheres. For our particular analyzer, $r_{1}=1.000^{\prime \prime}$ and $r_{2}=1.250 "$, so that the expected proportionality is given by $\mathrm{V}_{\mathrm{EA}}=0.45 \mathrm{E}$. We have measured the constant of proportionality by a method which we describe later in this chapter and find that

$$
\mathrm{V}_{\mathrm{EA}}=0.39 \mathrm{E} \text {. }
$$

The analyzer itself is fabricated from molybdenum; the choice of material for low energy experiments is critical because residual fields can deflect ion trajectories significantly. Molybdenum has been shown to be a desirable material because of its uniform surface potential when an electrostatic potential is applied. ${ }^{28}$ This contrasts with more common materials (including stainless steel) for which inhomogeneous grain size can lead to significant surface potential distributions. Copper is also a desirable material if the analyzer is made by electroforming under conditions where the grain size can be controlled closely; however, copper is subject to oxidation and spot-welded electrical connections cannot be made readily. 
An additional refinement we have made to our analyzer involves cutting a slot .250" wide through the back of the concave electrode and spotwelding $90 \%$ transmission mesh over the slot to restore the equipotential surface. This modification is quite important in nonreactive scattering experiments when backscattered ions are observed at energies much lower than the beam energy; under such circumstances with our modification, the undeflected primary beam can exit the analyzer rather than reflecting from the back surface of the analyzer and creating large numbers of background counts.

Significant advances in electron monochromator design at low energies have been made by Simpson and Kuyatt 25,26 and we have attempted to exploit some of these ideas for our analyzer. A particularly important feature for maximum transmission at low energies involves the use of virtual apertures, in which the beam size at the entrance plane of the analyzer is not determined by a slit, but rather by imaging a slit at a somewhat higher energy with a system of large aperture electrostatic lenses. One can either retard or accelerate the ions to a given energy for analysis which remains fixed throughout the experiment. Since $E_{a} / \Delta E$ is constant for a monochromator operating at some energy $E_{a}$, by making $\mathrm{E}_{a}$ constant, we also make $\Delta \mathrm{E}$ constant so that the data reflect the same $\Delta E$ bandwidth regardless of the laboratory energy. Clearly, the additional complexity of a virtual aperture systen requires careful design to recover the benefits inherent in such a configuration. Simpson and Kuyatt have developed a 
system of cylinder lenses for injecting and extracting electrons from a $180^{\circ}$ spherical deflector but we desired to use a $90^{\circ}$ deflector and space limitations prevented our choosing a comparable system of cylinder lenses in the injection stage. Instead, we have chosen a more compact system of aperture lenses which we discuss in the next section.

\section{Read lens injection system}

Because we desire to focus an object at variable energy onto the entrance plane of a $90^{\circ}$ analyzer, for which the focus is displaced from the physical boundary of the analyzer by one mean deflecior radius, a two-element lens is inappropriate because its focal properties are a unique function of the voltage ratio. Instead, we have chosen a three-element system of apertures for which focal properties depend on two voltage ratios and therefore we have the flexibility of focusing the beam cleanly to a given fixed plane as the energy varies by changing the potential on the center element only. Because the beam size at the entrance plane of the spherical analyzer determines the energy resolution, careful consideration of entrance aperture dimensions and ion optical properties of the injection system must be included in the apparatus design.

The Read lens system 29,30 of apertures which we have chosen is quite simple and consists of a three-element aperture lens in which the elements are spaced one aperture diameter apart. 
The potentials applied to each of the elements are different; in general, the first element is grounded so that $v_{1}$ is the nominal energy of the beam in the lab. $v_{2}$ is the potential applied to the second element; this is the potential which is varied to focus a variable energy beam to a fixed focal plane. $v_{3}$ is the potential applied to the third element of the Read lens. This potential determines the centcrline energy of the ions through the analyzer; the positive and negative potentials $\pm \mathrm{V}_{\mathrm{EA}} / 2$ applied to the concave and convex electrodes of the spherical analyzer respectively are measured with respect to $V_{3}$. Thus, if ions of energy $E_{\text {anal }}$ can pass through the analyzer, then the nominal energy of ions required to pass through the detector is given by

$$
E_{\text {ion }}=-V_{3}+E_{\text {anal }}
$$

with $V_{3}$ measured with respect to ground potential. Imhof and Read present data for the $v_{2} / v_{1}$ and $v_{3} / v_{1}$ ratios required to focus the object to the same image point as a function of mid-object and mid-image distances $P$ and $Q$ respectively. $P$ and $Q$ in their notation are measured in terms of aperture diameters; for our system, we chose an aperture diameter of $0.180^{\prime \prime}$ and in these units $P=4.0$ and $Q=4.0$. The Read lens has both a retarding and accelerating focus and we generally find the latter focusing conditions more desirable. Although no focusing curves were plotted by Imhof and Read for these values of $P$ and $Q$, examination of their plots demonstrates that for acceleration or retardation factors of three or less, 
the required second element potential is a convenient value i.e., seven or less. Because the transmission of the lens falls off dramatically if retardation in excess of a tenfold factor is required, the small factors required to operate our particular lens are indeed advantageous. Because the potentials required to satisfy the Read lens focusing criteria are quite empirical, we chose to determine the focusing voltages experimentally. All lens element potentials are referred to the centerline potential $V_{3}$; because one merely sweeps $v_{3}$ to perform energy analysis, only very small corrections to the Read lens potentials are required. Further discussion of the method for performing energy analysis is included in the next section.

The Read lens system is designed to focus the object formed by the detector entrance aperture onto the entrance plane of the spherical analyzer. The magnification of the optical system is important for determining analyzer resolution and the detector aperture dimension was chosen with this parameter in mind. Equally important and also determined by the aperture is the angular resolution of the detection system. The magnification of the Read lens system depends only on the mid-image and mid-object focal lengths $Q$ and $P$ respectively in the following manner:

$$
M=0.8 \frac{Q}{P} \text {. }
$$

For the dimensions we have chosen, the magnification of the detector aperture object at the spherical analyzer entrance 
plane is 0.8. Since the object dimension was chosen to be $.040 "$ ", the virtual slit dimension $w_{0}$ is thus 0.032". The energy resolution of the analyzer is then

$$
\frac{\Delta E_{1 / 2}}{E}=\frac{w_{0}}{2 r_{e}}
$$

with $r_{e}$ the mean radius of curvature of the analyzer; for our apparatus, $\mathrm{r}_{\mathrm{e}}=1.125 "$. We thus arrive at $\Delta \mathrm{E}_{1 / 2} / \mathrm{E}=.014$.

The real detector slit dimension determines the angular resolution of our system; the maximum angular range which the detector sees is defined by a ray passing through the collision center and the detector aperture boundary. The half angle thus defined is $0.75^{\circ}$, making the FWHM angular resolution $1.5^{\circ}$. The .040" detector defining aperture is viewed as the window of the optical system with the $0.120^{\prime \prime}$ slit considered the pupil. In a well designed optical system the entrance pupil should be located at one of the lens cardinal points to make the ion beam angle zero. While the Lagrange-Helmholtz law limits the beam pencil half-angles, the beam angle itself can be varied independently of this restriction. 31 Space limitations prevented placement of the lenses in this manner so we have added a field lens to our optical system between the Read lens and the deflector to restore the zero beam angle condition, thereby improving transmission through the analyzer. Kuyatt ${ }^{31}$ has indicated that a lens placed at an intermediate focus can correct for bad pupil placement. The field lens which we have constructed is basically an einzel lens fabricated from cylindrical lens elements. 
The construction of the lens is quite simple: the third element of the Read lens which is held at the scan or centerline potential is extended toward the detector with a cylinder lens. The element closest to the detector is also at the centerline potential and a third, intermediate cylindrical element is located at the intermediate focus. Consequently, the lens is an unipotential lens: we operate this lens in the accelerating mode by applying a negative potential with respect to the scan potential. Maximum transmission through the analyzer is determined by varying this potential.

The Read lens-field lens combination determines the angular and radial magnification of the detector aperture window onto the entrance plane of the analyzer; these quantities determine the filling factor for the analyzer. Kuyatt $^{31}$ has shown for an interelectrode gap of $w_{m}$, an injection half-angle of $\theta_{1}$ and a baseline resolution of $\Delta E / E_{0}$ that the following equation holds:

$$
\frac{W_{m}}{r_{e}}=\frac{\Delta E}{E_{0}}+\left[\theta_{1}^{2}+\left(\frac{\Delta E_{1 / 2}}{E_{0}}+\frac{\Delta E}{E_{0}}\right)^{2}\right]^{1 / 2}
$$

Kuyatt's discussion of the analyzer lineshape function for our assumed $\Delta E_{1 / 2} / E_{0}=.014$ and $\Delta E / E_{0}=.020$ yields $\theta_{1} \approx 5^{\circ}$. Consequently, a fairly large analyzer acceptance angle is conpatible with our entrance spot size of .032". A second parameter which Kuyatt discusses is the maximum $\Delta \mathrm{E}_{1 / 2} / \mathrm{li}_{0}$ allowed for which ions do not strike the electrodes; this value is .0138 , thereby placing a 1 imit on the virtual aperture size which one should allow in a spherical analyzer. We note 
that our deflector operates at this 1 imit.

A consideration of the Lagrance-Helmholtz law indicates that ions which exit the deflector at the maximum angle of $4.9^{\circ}$ and are accelerated by the post-analyzer lens by a factor of two will be collimated to $2.1^{\circ}$, well within the acceptance angle of the quadrupole mass filter.

3. Heddle Lens Extraction System

Ions which exit the spherical energy analyzer must be focused onto the entrance aperture of the quadrupole mass filter. Since the ions exit the analyzer at a variable energy and must be injected into the mass filter at a fixed energy with respect to the centerline potential, we have chosen a three element lens to perform this function. Once the potential at which mass analysis is performed has been selected, no further focusing is required because this potential floats on the scan potential. However, to provide us with the flexibility to mass analyze at various energies, a three element lens is necessary to focus ions onto the entrance aperture of the mass filter as a function of energy. This lens is designed in accord with the configuration proposed by Hedd $1 \mathrm{e}^{32}$ which employs cylinder lenses. The first element of the lens is held at the mean centerline potential of the ions through the analyzer. The second element of the lens can be made positive or negative with respect to the centerline potential and in analogy with the Read lens has a focus in either mode. In practice, we choose the mode which yields the greatest intensity at the detector; this mode frequently is retarding, 
but in either case, the applied voltage yielding the maximum intensity is within two volts of the centerline potential. The third element is electrically connected to the front plate of the quadrupole mass filter and is floated at the centerline potential of the mass filter. Mass analysis is generally performed at $10 \mathrm{eV}$ relative to the analyzer centerline and the appropriate potentials to this lens as well as the remaining detector lenses are supplied by Kepco modules divided across potentiometers.

An assembly diagram of the detector including the Read lens injection system, the analyzer, and the Heddle lens transport system is shown in Figure 8. Ions entering the detector pass through the aperture 20 which defines the detector angular resolution; the Read lens elements $\underline{18}$ and 19, fabricated from molybdenum, focus the beam and accelerate or retard the ions as required. Element $\underline{18}$ also forms the first element of the field lens which is completed by the cylinders $\underline{16}$ and $\underline{17}$. Ions are then focused into the analyzer comprised of convex electrode $\underline{8}$ and concave electrode $\underline{9}$. The electrodes themselves are spaced by precision sapphire balls of 1/8" diameter placed in appropriate reamed holes in the electrodes and mounting elements $\underline{10}-\underline{14}$. The Heddle lens elements $\underline{5}$ and $\underline{6}$ focus the beam exiting from the analyzer onto the quadrupole mass filter $\underline{3}$.

The analyzer and Read lens elements are assembled on the analyzer mount 7 which is secured to the detector chanber via a precision-machined bore and corresponding key on the mount. 
Figure 8. Energy analyzer assembly drawing

1. Rotating Detector Lid

2. QPMS Chamber

3. Quadrupole Mass Filter

4. Post Analyzer Lens Holder

5. Post Analyzer Ion Lens 1

6. Post Analyzer Ion Lens 2, 3

7. Analyzer Mount

8. Convex Electrode

9. Concave Electrode

10. Concave Electrode Holder

11. Analyzer Shielding Box

12. Electrode Mounting Plate - 1

13. Electrode Mounting Plate -2

14. Electrode Mounting Plate -3

15. Pre-analyzer Lens Mount

16. Field Lens Element A

17. Field Lens Element B

18. Read Lens 3

19. Read Lens Elements 1,2

20. Detector Aperture

21. Energy Analyzer Shielding Plate 


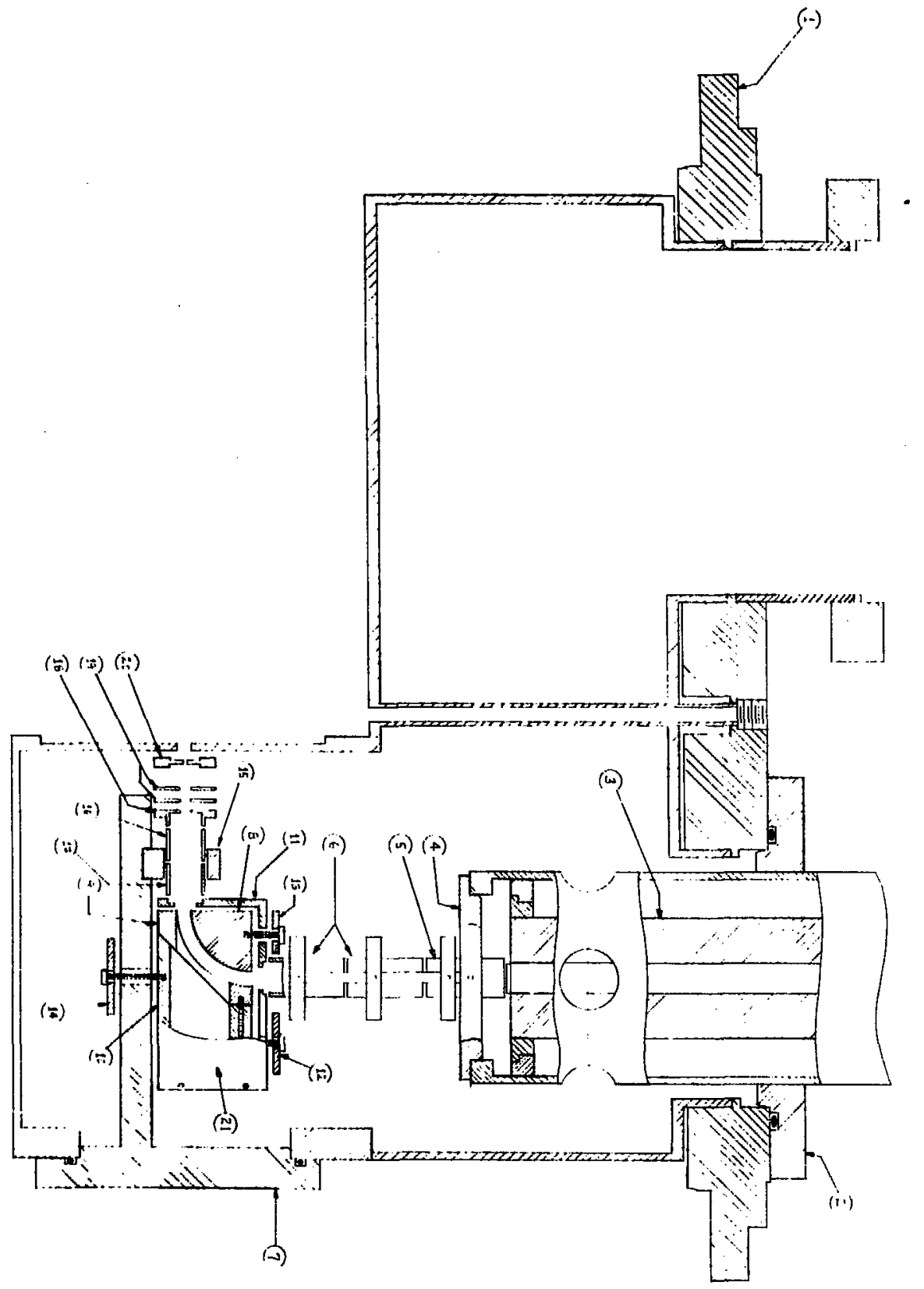


The mass filter and Heddle lens optics are mounted in the QPMS chamber $\underline{2}$ which keys into the detector chamber and is referenced to the accurately machined top surface of the rotating chamber.

Figure 9 shows the block diagram of the energy analyzer power supply interconnections. The centerline (scan) potential can be either positive or negative with respect to ground potential and the remaining power supplies are referenced with respect to this potential. In this manner, the Read and Heddle focusing requirements are established at a particular centerline potential and are satisfied over a reasonably broad range of scan values about the original voltage. Consequently, if the scan potential does not vary by more than $30 \%$ in either direction from the value used for the initial tuning, then energy analysis of the products can be accomplished merely by varying the scan potential with no corrections required for the Read and Heddle lenses.

Because both the potentials applied to the spherical analyzer electrodes and the centerline potential must be established to detect ions of a particular energy, independent variations of these two quantities should allow for a determination of the constant of proportionality between the energy of ions passed by the analyzer and the potentials applied to the electrodes. By taking an ion beam of fixed energy and applying different retarding and accelcruting centerline potentials in conjunction with corresponding electrode potentials, we have determined the relation between these two quantities as indicated in Eq. 18 . 
Figure 9. Block diagram of energy analyzer power supplies and interconnections. 


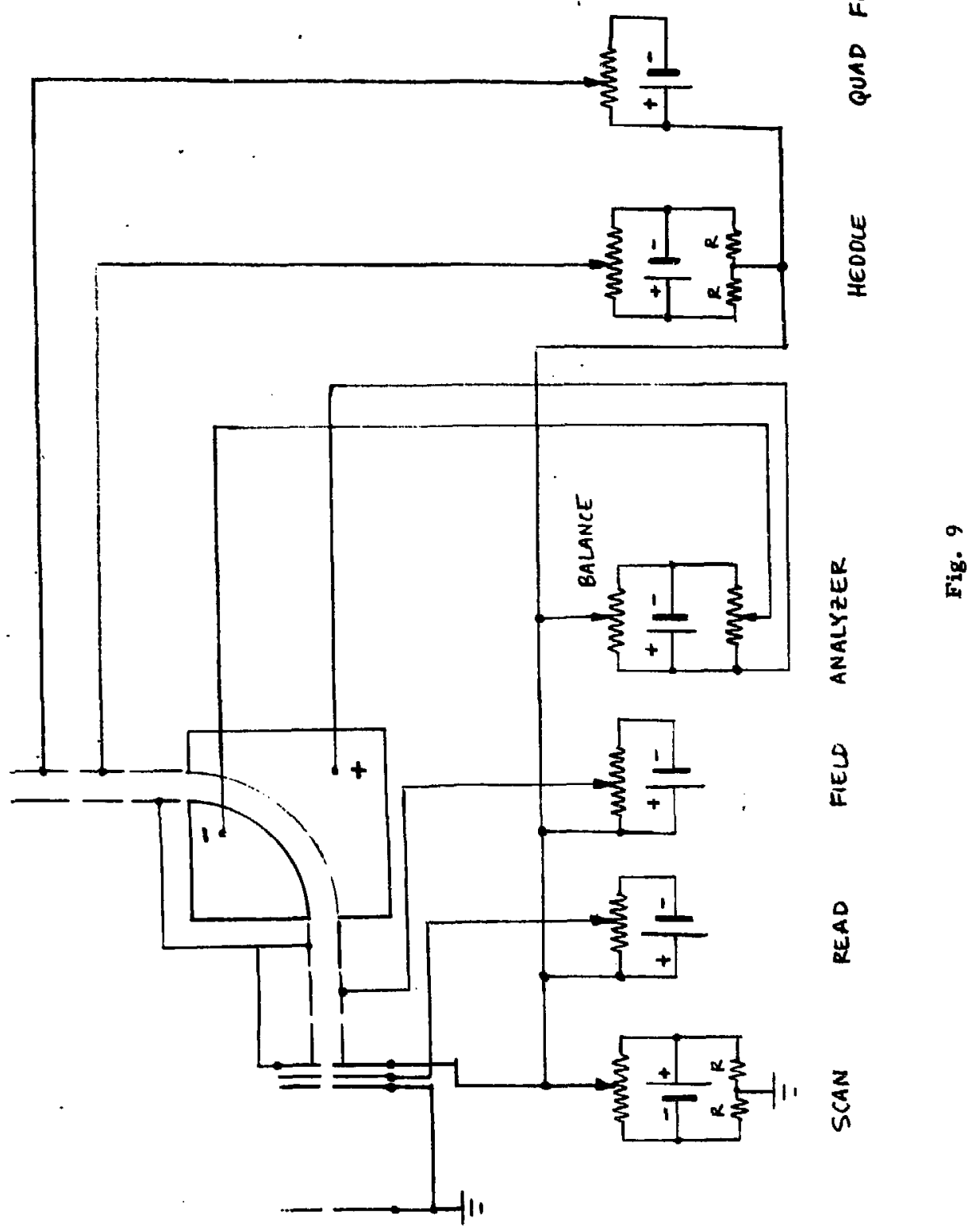




\section{Quadrupole Mass Filter}

The mass filter used in this apparatus is a radio frequency device originally developed by Paul ${ }^{33}$ and discussed by Wilson and Hartley. ${ }^{34}$ Our unit is like that described in Gentry's report: a portion of the $r f$ voltage is rectified to provide the DC potentials which make the filter mass selective. This rectifier is external to the main power supply and control unit and is mounted near the mass filter itself. The filter can be chosen to operate at any one of four different frequencies corresponding to the mass ranges $1-10,3-30,10-100$ and 30-300. Because mass resolution is a function of the number of oscillations which an ion makes in the filter, jons must be injected into the filter at as low an energy is consistent with minimal losses from space charge effects. The resolution question is confounded for high mass numbers by the low energies required for separation and the concomitant space charge difficulties. Since we have performed no studies of ions in this heavy regime, we have found that the filter works adequately for our purposes in spite of the relatively low operating frequencies compared to commercially available units. A recent significant advance reported by $F_{i t e} 35$ concerns the use of special dielectric materials which allow ions in the fringing field entrance and exit regions of the mass filter to experience only the net strong focusing $r f$ field when the ion is not deep within the mass filter. Such a field separation device prevents the loss of ions on trajectories which would be stable inside the filter but are defocusing in the fringing field region of the filter. This effect is 
especially important under the conflicting requirements of high resolution and high transmission encountered with heavy ions and the solution discussed by Fite holds great promise for improving mass filter performance by at least one order of magnitude.

The mass filter affords great advantages over conventional magnetic mass spectrometers because of compactness and the fact that resolution can be varied easily by changing the ratio of $r f$ and DC voltages over reasonably narrow limits. A further advantage derives from the relative insensitivity of the transmission of the filter as a function of ion energy. Careful design of the Heddle lens systen which injects ions into the mass filter allows ions which exit the analyzer at wide angles to be focused into a beam with divergence angle within the acceptance angle of the mass filter; under these circumstances very high transmission through the mass filter can be achieved with good resolution.

\section{E. Scintillation Ion Detector}

1. Genera1 Remarks

- After ions have passed through the mass filter, they must be recorded and communicated to the experimenter in a useable form. The most efficient means of recording low level signals which result from discrete events is to employ digital counting techniques for recording the individual events. The particular ion detector which we have chosen converts ion pulses to photomultiplier tube pulses which can be translated into digital signals suitable for counting with conventional 
pulse counting equipment. The device we use is quite sensitive and has the advantage of mechanical simplicity and reliable, trouble-free operation.

Particle detectors which convert events into visible light pulses are referred to as scintillation derectors; the design we use is due to Daly ${ }^{36}$ and has become a standard in molecular beam detectors. 37 Figure 10 shows the construction of the scintillation detector which we employ in our apparatus. Mass selected ions exiting the mass filter in the vertical direction must be collimated into a bean suitable for the ion detector; the cylindrical lens elements at the bottom flange of the detector accelerate the beam to $2 \mathrm{kV}$ and focus the beam. The first and third lens elements are held at $-2 \mathrm{kV}$ while the middle element is generally held at $-600 \mathrm{~V}$ for sharp focusing of the beam. The fast ion beam then strikes the aluminum coated stainless steel cathode $\underline{8}$ to which a $-25 \mathrm{kV}$ potential is appiied; maximum secondary electron emission occurs for incidence angles as far from normal as possible. Ions striking the aluminum cathode eject secondary electrons ( $-3-5$ per ion) which are accelerated away from the cathode by the same potential which attracts the positive ions. These accelerated electrons strike a sheet of $.005 "$ plastic scintillation material (NE 102, Nuclear Enterprises, Inc., San Carlos, CA) which has been epoxied to a $3 \mathrm{~mm}$ quartz disc. The front face of the scintillator is vacuum coated with $1000 \AA$ of aluminum to reduce stray light which may enter the photomultiplier tube and to insure that the scintillator face is grounded to drain off the charge resulting from the secondary electrons. 
Figure 10. Scintillation detector assembly drawing. This drawing is specifically for the EMI 9824B type detector installed on apparatus "Charlie".

1. Detection Chamber

2. PMT Chamber

3. PMT Housing

4. PMT Housing Endcap

5. Cathode Flange

6. High Voltage Protection Spool

7. High Voltage Protection Shield

8. Ion Target

9. Sliding Sea1

10. Anti-corona Ball

11. Shielding Sleeve

12. RG 213 A/U HV Cable

13. NE 102 Plastic Scintillator Sheet

14. Quartz Disc

15. PMT Scintillator Mounting Nut

16. EMI 9824B Photomultiplier Tube

17. EMI Magnetic Shielding

18. EMI PMT Socket B14B

19. Dynode Chain Holder

20. Spacer

21. SHV 1702-1 High Voltage Connector 


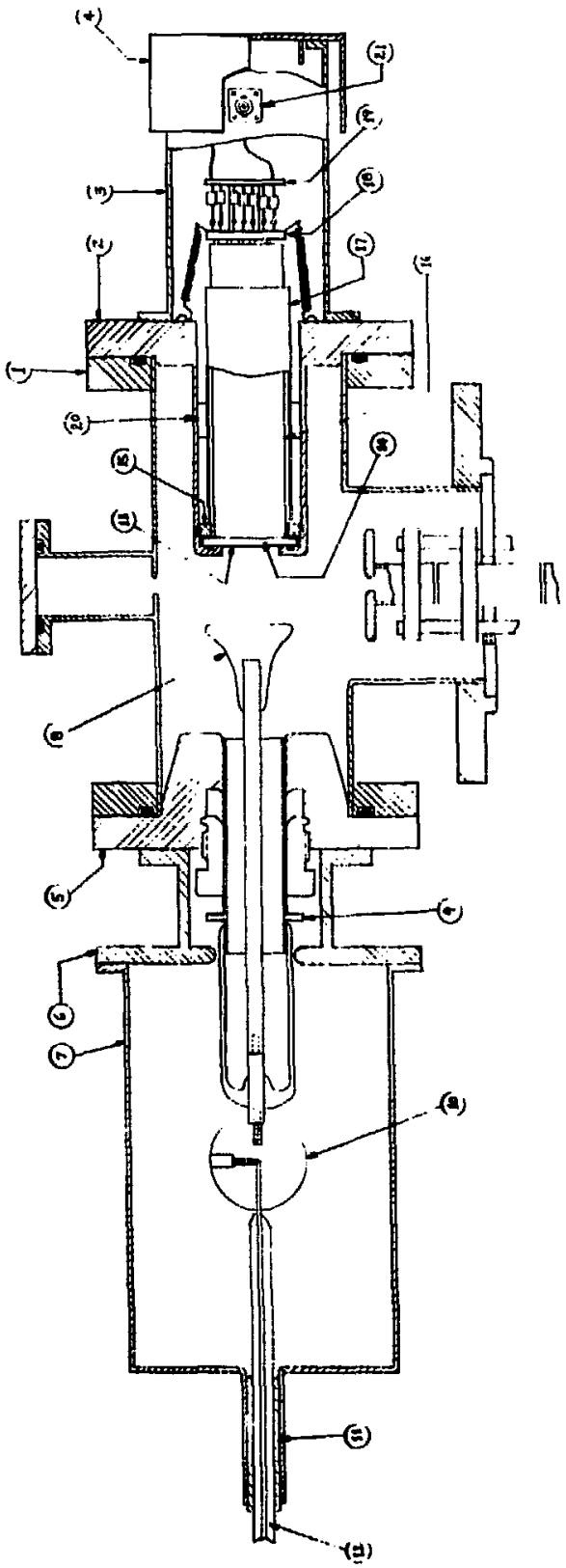

웅 
The photomultiplier tube is not contained in the vacuum envelope; the quartz-scintillator disc itself maintains a vacuum seal against the window of the PMT chamber 2 . A piece of 1/16" diameter indium solder joined into an 0 -ring gasket of the appropriate diameter provides the desired vacuum seal; the gasket is compressed with a scintillator mounting nut 15 made from brass which threads into the PMT chamber with a special wrench. The high energy electrons result in photon emission from the plastic scintillator; six to eight photons are emitted per incident electron and the average pulse risctime for a photon generated in the scintillator is two nsec. Photons emitted by the scintillator are coupled to a photomultiplier tube and the anode pulse is processed by conventional nuclear counting instrumentation. Two different scintillation detectors have been fabricated which differ in the choice of photomultiplier tubes; these will be discussed in separate sections below.

Care must be taken in the fabrication of the scintillation detector with regard to the high voltages required in its operation. Al1 detector components are carefully machined and polished to remove all sharp edges, small radii corners, burrs, scratches, and nicks. The high voltage cathode feedthrough $\underline{9}$ embcdies a Wilson seal to allow the cathodescintillator distance to be optimized. A special large radius anticorona ball $\underline{10}$ is employed to prevent arcing outside the vacuum envelope and a high voltage protection shield $\underline{7}$ prevents the experimenter from exposing himself to lethal voltages. The power supply which provides the high voltage is constructed 
from a special module salvaged from Lawrence Berkeley Laboratory, filtered with a .05 Mfd capacitor, energized with a Variac and carefully interlocked for safety.

The operation of any detector employing high voltages requires care in insuring that arcing which might result from surface imperfections or ionization of residual gas is minimized. The scintillation detector components were carefully polished and the entire unit leak checked pricr to operation. A carefully constructed unit is capable of very efficient operation and specific operating characteristics are indicated in the two sections which follow.

\section{EMI Detector}

The detector which was designed first includes an EMI 9824B phototube, a 1" diameter tube with a bialkali cathode and 11 box and grid dynodes fabricated from $\mathrm{Be}-\mathrm{Cu}$. This particular configuration does not yield extremely sharp pulses and the overall gain of the tube at $1260 \mathrm{~V}$, chosen to optimize signal to noise ratio, is not extremeiy high. This tube was chosen because of its very low dark current (.02 na) and low price. Because of the additional mechanical complexity required to operate a phototube with the photocathode at high negative potential resulting from the requirement of floating the magnetic shielding at the cathode potential, we have elected to use a negative ground design. This design allows the cathode and magnetic shield to be at ground potential while the anode is at high positive potential. Zener diodes between the cathode and first dynode maintain a constant 
voltage drop regardless of the overall applied potential; a linear dynode chain documented in the Engineering Notes for this apparatus supplies the necessary voltage increments to be applied to the tube and bypass capacitors decoupling the last two dynodes from ground allow the dynode roltages to remain constant during the transit of a high current pulse in the last stages of the tube. AC coupling of the output pulse results in a time constart of 500 nsec, limiting the output pulse width; this time constant reduces the dynamic range of the detector but counting rates of $2 \mathrm{MHz}$, seldom realized in practice, can be accommodated readily. The anode of this tube is reverse terminated with a $51 \mathrm{ohm}$ resistor to reduce spurious signals arising from reflections at the amplifier input. This feature reduces output pulse height by a factor of two, but multiphoton pulses arising from ions have pulse heights which exceed $200 \mathrm{mV}$, in contrast to dark current pulses of amplitude $5 \mathrm{mV}$, making discrimination quite easy. This detector has been in operation for over a year and performs quite well with background counting rates in the range of $2-3$ counts per second.

3. RCA Detector

For the low energy apparatus described in this report, we have fabricated a scintillation detector from a 2" diameter photomultiplier tube, an RCA 8575. This particular tube is a standard for nuclear physics instrumentation with excellent risetime characteristics resulting from a linear-focused dynode structure. This tube consists of a bialkali photocathode 
with quantum efficiency of $31 \%$ and 12 dynodes fabricated from $\mathrm{Be}-\mathrm{Cu}$. As with the EMI tube, we have elected to use a positive high voltage supply for mechanical simplicity; the anode time constant is $250 \mathrm{nsec}$, limiting counting rates to $4 \mathrm{MHz}$. Reduction of the value of the coupling capacitor consistent with the reverse termination resistor requirement of $51 \mathrm{ohms}$ will yield a lower value for the time constant if higher counting rates must be accommodated.

The dynode chain used for this tube is documented in the Engineering Notes and is essentially that recommended by RCA. The potential drop between the cathode and first dynode is four times the drop between other pairs of dynodes in the tube. For a typical operating voltage of 2100 volts, the average interdynode potential difference is 140 volts with 560 volts across the cathode and first dynode. The last four dynodes are capacitively decoupled and the anode is AC coupled and reverse terminated. The pulse height from the tube under these conditions is sufficiently high that an amplifier is not required before the discriminator. The output from the phototube is fed to a LeCroy 321B discriminator with the threshold set to the minimum value of $100 \mathrm{mV}$. Under these circumstances, the background counting rate with no ions incident on the detector is 0.5 cps.

Daly $y^{36}$ has measured the pulse height distribution for a scintillation detector and the results indicate that the individual distributions corresponding to ejection of single and multiple photoelectrons from the cathode overlap and cannot be resolved. Consequently, a pulse height discriminator 
which removes low amplitude noise pulses also removes a small fraction of signal pulses. The solid state detector described by Gentry was employed to overcome this limitation because the high resolution inherent in such a detector allows one to discriminate cleanly between events differing in the numbor of secondary electrons emitted. However, such a detector is more complex than a scintillation detector and, in fact, operating difficulties with the solid state detector motivated us to abandon it in favor of the phototube detector. The loss in resolution and concomitant noise increase has not been a serious problem, but recent technical advances in phototube design yield instruments which can rival the solid state detector in performance. The RCA 8850 phototube possesses the same basic structure as our 8575 tube with one simple modification: the $\mathbf{8 8 5 0}$ first dynode is fabricated from gallium phosphide, a material with excellent secondary emission characteristics which preserves the information in the photon pulse regarding the number of photoelectrons emitted by the cathode. The 8850 tube fits in the same socket as the 8575 and its dynode chain characteristics are also compatible; a future modification of this detector then should be replacement of the 8575 tube with the 8850 with a resulting tenfold decrease in background noise. 


\section{References, Chapter II}

1. H. Udseth, C. F. Giese and W. R. Gentry, Phys. Rev. A $\underline{8}$, $2483(1973)$.

2. F. H. Field, Acct. Chem. Res. 1, 42 (1968).

3. M. L. Vestal, C. R. Blakley, P. W. Ryan, and J. H. Futre11, Rev. Sci. Instrum. 47, 15 (1976).

4. M. Chiang, Ph.D. dissertation, University of California, Berkeley (1967).

5. L. Wâhlin and M. Menzinger, Rev. Sci. Instrum. 40, 102 (1969).

6. K. R. Spangenberg, "Electrostatic Electron Optics", in Vacuum Tubes (McGraw-Hill, New York, 1948), Chapter 13.

7. C. Lu and H. E. Carr, Rev. Sci. Instrum. 33, 823 (1962).

8. C. A. McDowe11, ed., Mass Spectrometry (McGraw-Hill, New York, 1963).

9. F. M. J. Pichanick, "Ion Spectrometers", in Methods of Experimental Physics, Vol. 4A, V. W. Hughes and H. L. Schultz, eds. (Academic, New York, 1967), p. 363.

10. M. L. Vestal, C. R. Blakley, P. W. Ryan, and J. H. Futre11, in Advances in Mass Spectrometry, Vol. 6, J. R. West, ed. (Applied Science Publishers, London, 1974), p. 781.

11. M. L. Vestal, Ph.D. dissertation, University of Utah, 1975.

12. P. Grivet, Electron Optics (Perganon, London, 1965).

13. A. Kantrowitz and J. Grey, Rev. Sci. Instrum. 22, 328 (1951).

14. G. B. Kistiakowsky and W. P. Slichter, Rev. Sci. Instrum. 22, 333 (1951). 
15. R. Campargue, Adv. Appl. Mech. Suppl. 3, "Rarefied Gas Dynamics", vol II, ed. J. H. deLeeuw (Academic, New York, 1966), p. 279 .

16. U. Bossel, F. C. Hurlbut, and F. S. Sherman, Adv. Appl. Mech. Supp1. 5, "Rarefied Gas Dynamics", vol II, ed. J. H. deleeuw (Academic, New York, 1969 ), p. 945.

17. H. Ashkenas and F. S. Sherman, Adv. Appl. Mech. Supp1. 3, "Rarefied Gas Dynamics", vol. I I, ed. J. H. deleeuw (Academic, New York, 1966), p. 84.

18. J. B. Anderson and J. B. Fenn, Phys. Fluids $\underline{8}, 780$ (1965).

19. F. P. Tully, Y. T. Lee, and R. S. Berry, Chem. Phys. Lett. $\underline{9}, 80$ (1971).

20. F. P. Tully, Ph.D. dissertation, The University of Chicago, 1973.

21. E. W. Becker, K. Bier, and H. Burghoff, Z. Naturforsch. 10a, 565 (1955).

22. J. A. Simpson, Rev. Sci. Instrum. $\underline{32}, 1283$ (1961).

23. P. Marmet and L. Kerwin, Can. J. Phys. 38, 787 (1960).

24. E. M. Purce1l, Phys. Rev. 54, 818 (1938).

25. C. E. Kuyatt and J. A. Simpson, Rev. Sci. Instrum. 38, 103 (1967).

26. C. E. Kuyatt and E. W. Plummer, Rev. Sci. Instrum. $\underline{43}$, 108 (1972).

27. W. Steckelmacher, J. Phys. E: Sci. Instrum. 6, 1061 (1973).

28. G. J. Schulz, Rev. Mod. Phys. 45, 378 (1973).

29. R. E. Imhof and F. H. Read, J. Phys. E: Sci Instrum. 1, 859 (1968). 
30. F. H. Read, J. Phys. E: Sci. Instrum. 2, 679 (i969).

31. C. E. Kuyatt, Electron Optics Notes, unpublished.

32. D. H. O. Heddle, J. Phys. E: Sci. Instrum. 2,1046 (1969).

33. W. Paul and H. Steinwede1, 2. Naturforsch. 8a, 448 (1963).

34. K. R. Wilson, University of California, Lawrence Radiation Laboratory Report UCRL-11605 (1964) .

35. W. L. Fite, Rev. Sci. Instrum. 47, 326 (1976).

36. N. R. Daly, Rev. Sci. Instrum. 31, 264 (1960).

37. Y. T. Lee, J. D. McDonald, P. R. LeBreton, and D. R. Herschbach, Rev. Sci. Instrum. 40, 1402 (1969). 


\section{Operation of the Apparatus}

The procedure which one follows to obtain enersy and angular scans with this low energy apparatus is, in general, quite simple, particularly since one is interested primarily in relative differential cross sections. In this chapter, we will sketch the procedure which is employed to get the apparatus under vacuum, obtain a satisfactory ion beam, and detect product species of the appropriate mass and energy.

Because of the prohibitive cost of liquid nitrogen, the apparatus is evacuated by the diffusion pumps only when the machine is in use. When the apparatus is to be shut down for an extended period or overnight, the three gate valves to the diffusion pumps are closed. With the apparatus closed off overnight, the pressure rises to about 500 microns, but pumpdown to $5 \times 10^{-7}$ torr can be achieved within 30 minutes from this condition. In order to insure maximum cleanliness, the detector aperture heater remains on at all times to keep insulating deposits from building up.

A11 mechanical punps backing diffusion pumps are protected with solenoid valves which are energized by the vacuum interlock system. Any condition which results in power loss to either of the two main chamber diffusion pumps (e.g., high foreline pressure, overtemperature, or restricted watcr flow) closes the solenoid valves to all three pumps. Conversely, a condition which terminates power to the ion source diffusion 
pump only results in energizing the solenoid valve for that pump. The solenoid valves thus prevent mechanical pump oil from backstreaming to the ion optics through the cold diffusion pumps.

After the apparatus has been evacuated and leaks have been eliminated, the filament is heated and the appropriate potentials applied to the ion source mass spectrometer optical elements; the appropriate values have been indicated in Chapter II. It is generally useful to leave power supply potentiometers set to the values used for the previous day's experiments and using the on-off toggle switches to energize the supplies. At this point the filament emission current should be measured by connecting the electrometer to the external connection for the ion source anode; acceptable values are in the range 3 to $6 \mathrm{ma}$. The gas to be admitted to the ion source is now bled into the inlet system through a Granville-Philips variable leak valve; the ion source vacuum chamber should not exceed $5 \times 10^{-5}$ torr at this point. A beam current should now be detectable on the main chamber Faraday cup and the beam can be focused onto this detector. After the beam has been found on the main chamber Faraday cup, it can be passed through the detector energy analyzer, quadrupole mass filter, and scintillation ion detector. The energy characteristics of the ion beam can be determined with the detector in this manner, then: the inass of the ion beam is set on the quadrupole mass filter control, the scintillation detector is turned on and the energy analyzer potentials and corresponding Read lens element values are set. 
The ion beam is characterized in energy and angular space and the $20 \%$ and $50 \%$ beam profiles are noted. Frequently, focusing of the ion source mass spectrometer can result in beam characteristics much improved over those initially determined. We have noted in general for parent ions such as $\mathrm{N}_{2}{ }^{+}$and $\mathrm{H}_{2}{ }^{+}$, that $\mathrm{FWHM}$ energy spreads of $0.2 \mathrm{eV}$ nave been obtained while for $\mathrm{N}^{+}$and $\mathrm{F}^{+}$generated from $\mathrm{N}_{2}$ and $\mathrm{NF}_{3}$, FWHM energy spreads of $0.8 \mathrm{eV}$ are typical.

After a primary ion beam of satisfactory quality has been achieved, it is crossed by a neutral beam generated by establishing a nozzle stagnation chamber pressure of 50 to 80 torr; under these circumstances the main chamber pressure rises to $2-4 \times 10^{-6}$ torr. The desired reaction product mass is set on the mass filter and the peak shape is determined by scanning the mass dial through the product mass number while noting the signal level on a Mech-Tronics counting rate meter. The mass filter coarse bias control should be adjusted to insure that good resolution between peaks differing by one amu is achieved: this is crucial for hydrogen abstraction reactions for which the product mass number differs from the primary ion beam mass by one unit.

Once the detector has been tuned for the desired product mass, one must determine the energy and angular range over which data must be collected. A consideration of the Newton diagram for the reaction under study with special attention to the limits on $Q$, the translational exoergicity, as a function of initial kinetic energy should determine the data coilection range. Data can be collected in two ways: 
1) The scan method, in which data are acquired at a fixed laboratory angle and the detector analyzer is swept through the desired energy range. This method is the one most amenable for computer controlled data collection with signal averaging.

2) The cut method, in which the energy analyzer is set for a particular energy and data are collected by rotating the detector over a range of laboratory scattering angles.

The latter method been used for our experimental studics to date. When one performs an experiment with this technique, the highest laboratory energy at which data are to be collected is determined and the scan and analyzer potentials are set appropriately. The Read and Heddle lenses are tuned on the primary beam energy and the first cut is taken. Output pulses from a Lelroy $321 B$ discriminator are sent to a Harshaw scaler which is controlled by a preset timer and teletype interface. After the scaler has counted pulses for a predetermined period of time, the counter halts and the data are printed out (and punched if desired) on an ASR-33 teletype. The next angle is selected and the process repeats until the cut is completed.

Because significant background counts are generated by chemical reactions which do not originate from the crossed beam intersection volume, a procedure has been developed to correct for these reactions. One cut is performed with the crossed beam on and the data are recorded; a second cut with the same detector potentials is then recorded but with gas 
leaked in through a valve located 1 " below the nominal centerline with respect to which the beam source is aligned; the pressure in the main chamber is adjusted to match the value observed with the neutral beam on. The background counts thus generated are recorded and subtracted from the corresponding values obtained with the neutral beam source on.

The scan potential is reduced and experimental data are then acquired for 10 to 15 cuts, the energy increments between successive cuts chosen as required to cover the desired range. We have found it sufficient to vary the centerline (or scan) potential only as data for laboratory energies of successively lower values are measured. The data as output on the teletype are processed as indicated in the next chapter.

The general energy range of this machine is well described by our initial design goal of 1 to $10 \mathrm{eV}$. We have detected product ions at lab energies as low as $0.15 \mathrm{eV}$; the lowest energy experiment for which a complete contour map has been generated is the $\mathrm{N}^{+}\left(\mathrm{H}_{2}, \mathrm{H}\right) \mathrm{NH}^{+}$system at a beam energy of $1.4 \mathrm{eV}$. Products of this reaction have been measured at $0.8 \mathrm{eV}$ readily; the fairly large energy spread of the $\mathrm{N}^{+}$primary ion beam made lower energy experiments seem to be of marginal value. In summary, the apparatus capability appears to be 1 imited not by the ability to produce high quality low energy beams, but rather by the transmission of very low energy ions $(\leq 0.5 \mathrm{eV})$ through the detector. 


\section{Kinematic Analysis}

Although detection of the products of a molecular colitision occurring in crossed molecular beams is accomplished in the laboratory system of coordinates, one desires ro understand the dynamics in terms of a coordinate system which moves with the center of mass (c.m.) of the colliding system. This transformation can be accomplished uniquely only in the case where both energy and angular distribution measurements of the scattered products are determined. A consideration of the nature of the kinematic problem is of necessity since Jacobian transformations relating one set of coordinates to another frequently distort intensities in one set of coordinates, making products appearing at certain sets of $1 a b$ angles and velocities (those near the c.m.) appear more intense than they actually are. ${ }^{1}$ The classic example of this problem occurs in the early studies of alkali atom - alkali halide exchange reactions ${ }^{2}$ in which preliminary observations of product angular distributions indicated that the reaction products were extremely highly excited internally, with nearly 908 of the total energy of the system in vibrational and rotational excitation. These erroneous conclusions were made because improper transformation between the c.m. and lah coordinate systems failed to note the Jacolian singularity at small c.m. recoil velocities which enhanced the lab intensities at these points; early conclusions were thus 
based upon lab observations which superficially indicated that products were preferentially formed with low c.m. recoil velocities. 3

The kinematic problem has been studied on several occasions ${ }^{1,4-7}$ and the nature of the transformation is well understood; some of the details appropriate to the ion beam collision cell apparatus in this laboratory have been discussed by Gentry. ${ }^{8}$ Our discussion will be somewhat more general initially, by discussing specifically the transformations used in this $1 a b$ and the manner in which data are displayed. Figure IV-1 presents a velocity vector diagram, or Newton diagram for the general A + BC collision which provides a geometrical method for relating lab velocities and scattering angles $\underset{\sim}{\mathbf{v}}$ and $\theta$ to their corresponding c.m. values $\underset{\sim}{\mathbf{u}}$ and $\theta$. The beam velocity vectors $\underline{v}_{A}$ and ${\underset{\sim}{B C}}_{B C}$ appropriate for an $A+B C$ collision intersect at $90^{\circ}$ and define the relative velocity vector ${\underset{\sim}{\mathrm{A}}}_{\mathrm{A}}-\underline{\mathrm{v}}_{\mathrm{BC}} \equiv \underline{v}_{\mathrm{rel}}$. The direction and velocity of the center of mass of the system are determined by the conservation of linear momentum; the vector $\underset{\sim}{C}$ divides ${\underset{\sim}{r e l}}_{\text {in }}$ inverse proportion to the masses of $\mathrm{A}^{+}$and $\mathrm{BC}$ :

$$
\underline{u}_{A}=-\frac{M_{B C}}{M_{A}+M_{B C}} v_{r e l} \text { and }{\underset{\sim}{B C}}_{B C}=\frac{M_{A}}{M_{A}+M_{B C}} v_{r e l} \text {. }
$$

An observer moving away from the laboratory origin in the direction $\theta_{\mathrm{cm}}$ with speed $|C|$ would see the reactants $A$ and $B C$ approach along a line and the products retreat along a line. The angle between these two lines, the initial and final relative velocities, is the c.m. scattering angle. In Figure IV-1, 
the details are shown explicatly. If we desire to detect products with lab velocity $y$ at angle 0 , we note that the corresponding c.m. velocity $\mathrm{u}$ is given by

$$
\underline{u}=\underline{v}-\underline{C} .
$$

The transformation from the lab system to the c.m. coordinate system merely involves translation of the laboratory origin through $\subseteq$ followed by counterclockwise rotation through $\theta_{v} \cdot{ }^{7}$ The 1 inear transformation relating the lab coordinates $\left(v_{x}, v_{y}\right)$ to the c.m. coordinates $\left(u_{x}, u_{y}\right)$ is written in matrix form as follows:

$$
\left(\begin{array}{l}
u_{x} \\
u_{y}
\end{array}\right)=\left(\begin{array}{cc}
\cos \theta_{v} & \sin \theta_{v} \\
-\sin \theta_{v} & \cos \theta_{v}
\end{array}\right)\left(\begin{array}{c}
v_{x}-c_{x} \\
v_{y}-c_{y}
\end{array}\right)
$$

Clearly, if one desires to determine the locus of c.m. recoil vectors $\underset{\sim}{u}$, then the convolution of the measured distribution $\mathbf{v}$ with $\mathrm{C}$ must be quite small; this situation can only be obtained when experiments are performed with beams with very narrow velocity distributions.

Another point of interest in Figure 11 concerns the locus of points in the laboratory associated with products whose recoil speed is constant. Clearly, these loci will be given by spheres with centers at the origin of the c.m. coordinate system and with radii given by the desired valuc of $u$. The elastic circle marked on Figure 11 describcs the locus of A particles in the plane of the beams scattered with no net change in the value of $u_{A}$. Corresponding circles 
Figure 11. Newton diagram showing geometrical relations

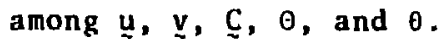




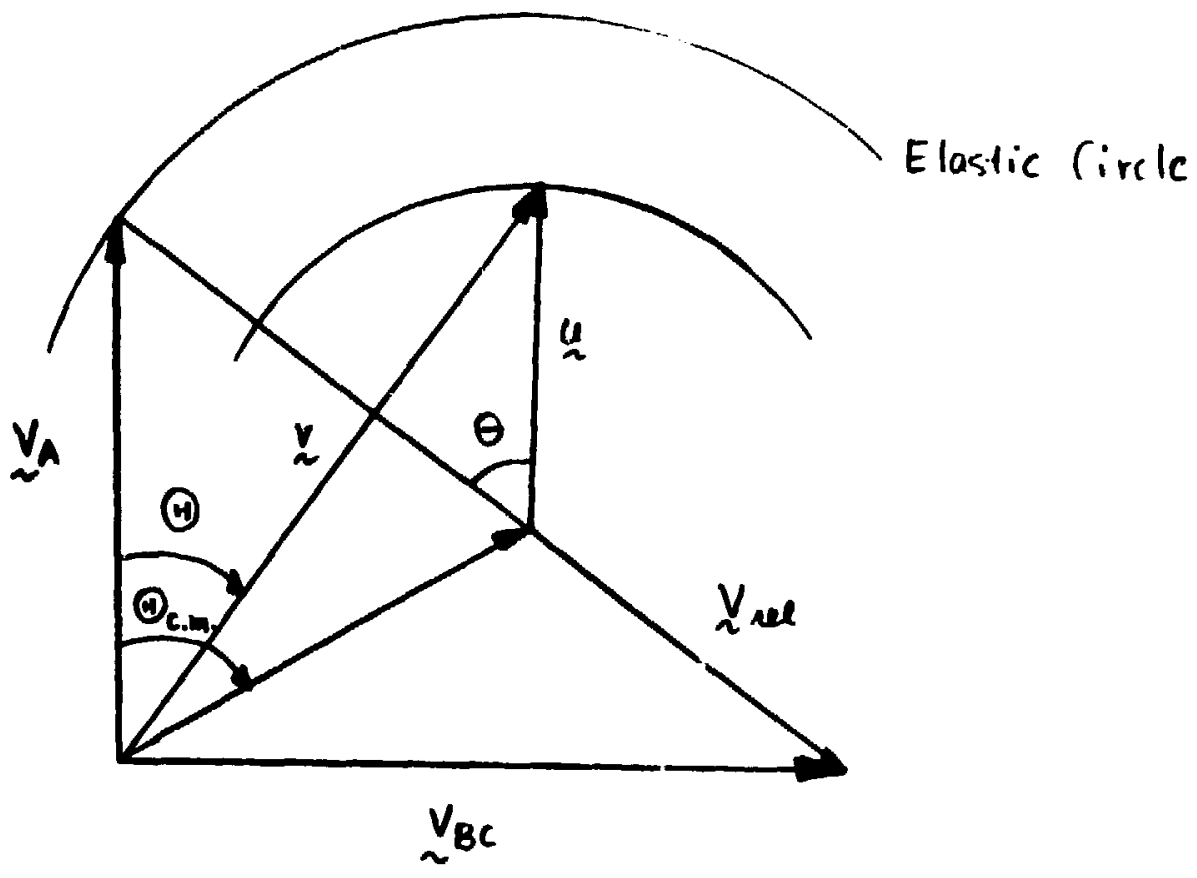

Fig. 11 
for inelastic or reactive cases can be drawn yuite readily.

We now consider the important question of intensity transformations between the two cooruinate systems. The nature of the transformation has been discussed in great detail and an especially valuable reference is the paper by Warnock and Bernstein. ${ }^{6}$ We will first discuss the geometrical aspects of the transformation followed by a discussion of the Cartesian representation used by many workers (and described by (ientry) as formally proposed by Wolfgang and Cross. ${ }^{9}$

An important fact to keep in mind in considering intensities in the c.m. and lab systems is that product flux, measured in units of particles per unit area per unit time, is the quantity which is conserved in the transformation between coordinate systems moving with respect to one another. Product detectors do not aiways measure flux and this should be kept in mind; electron bombardment ionization detectors, for example, measure product number density, whereas the energy analyzer detectors used on ion-molecule machines such as those in this laboratory are flux detectors.

Let us now consider the general naturei of the Jacobian appropriate to reactive scattering. Figure 12 compares the appropriate elemerics of volume in the c.m. and lab systems. If the detector, at some laboratory angle $\theta$ subtends a solid angle d $\Omega$ defined by the surface dS and volume element components $d v$, do, d $\Phi$ then the same dS is appropriate to the c.m. system and the flux through this surface is conserved. Equating elements of volume in the c.m. and lab systems, we have

$$
v^{2} d v d \Omega=u^{2} d u d w \text {. }
$$


Figure 12. Newton diagrams and volume elements. Upper panel: Lab coordinate system showing appropriate dimensions on volume element. Lower panel: corresponding element in c.m. system. 
94
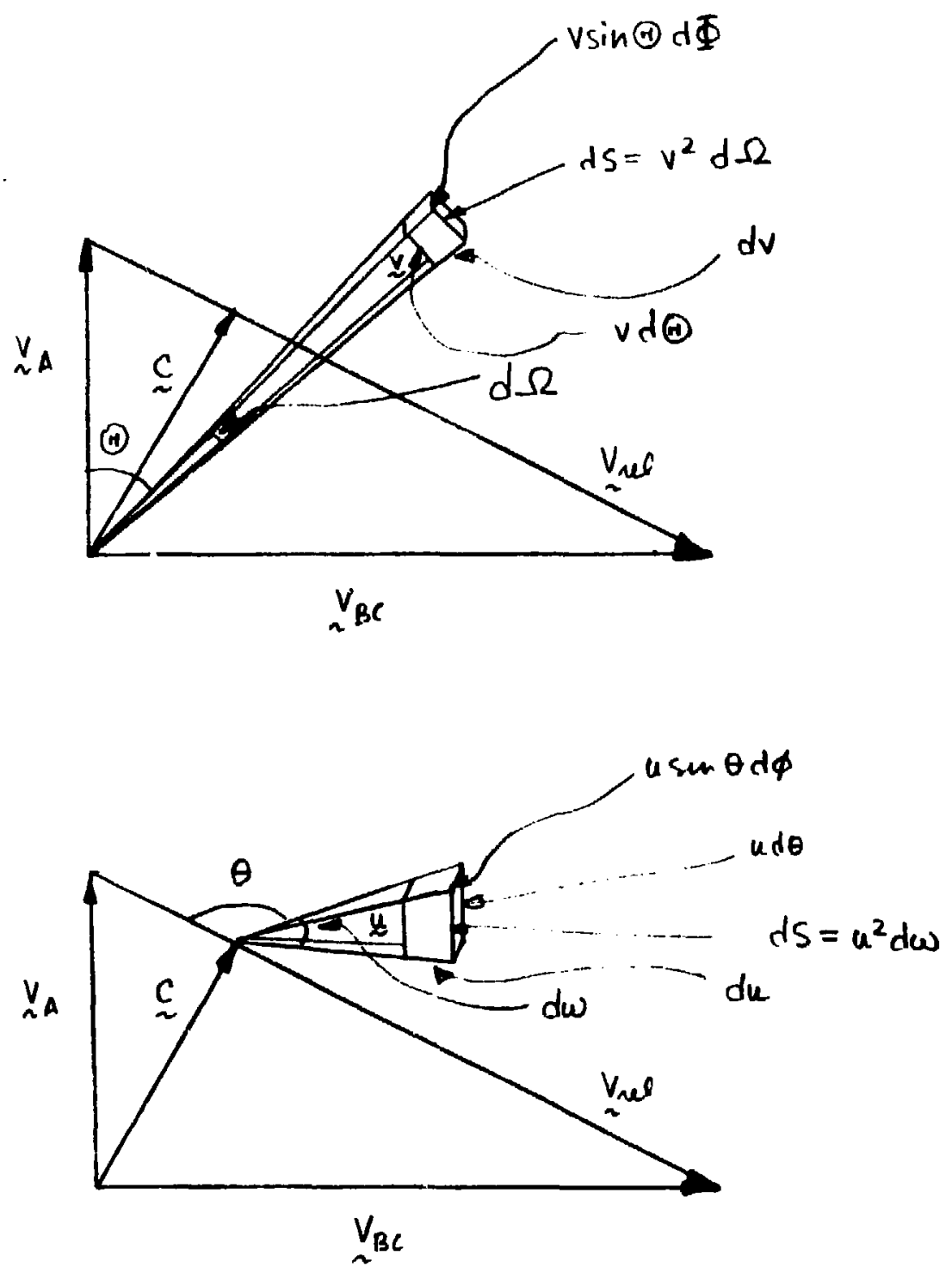

Fig. 12 
Equivalently, we can compute this element of volume as follows:

$$
v^{2} d v \sin \theta d \theta d \phi=u^{2} d u \sin \theta d \theta d \phi
$$

as indicated by Gentry. ${ }^{8}$ Defining the distribution of particle flux in the laboratory range $\theta$ to $\theta+\mathrm{d} \theta, \phi$ to $\phi+\mathrm{d} \phi$ and $v$ to $v+d v$ by $I_{L}(\theta, v)$ and the corresponding distribution in the c.m. system by $I_{\text {c.m. }}(\theta, u)$, we can write

$$
I_{L}(\theta, v) d v d \Omega=I_{c . m .}(\theta, u) d u d \omega
$$

or

$$
\left.I_{c . m .}(\theta, u)=I_{L} i v, v\right) \frac{d v d \Omega}{d u d w} .
$$

The equality of c.m. and lab volume elements enables us to write this expression as

$$
I_{c, m .}(\theta, u)=\frac{u^{2}}{v^{2}} I_{L}(\theta, v)
$$

or

$$
I_{L}(\theta, v)=\frac{v^{2}}{u^{2}} I_{c, m .}(\theta, u)
$$

We see, therefore, that lab intensities near the system's c.m. are weighted disproportionately because of the divergence as u becomes small.

Before discussing a representation for data which removes the distortion arising from the $1 / u^{2}$ singularity, we shall derive the Jacobian for a more specialised case in which only a single recoil speed in the c.m. system is allowed, as in c1astic scattering. Since the Jacobian appropriate to this 
case can be quite different from the one derived above for the case of many closely spaced recoil speeds, we show the derivation here and indicate its range of validity. Lxamination of Figure 13 shows that the detector subtends a solid angle $\mathrm{d} \Omega$ in the laboratory system. The locus of recoil vectors u with fixed magnitude maps out a sphere $S$. For a given laboratory velocity vector $v$, vectors $\underline{w}$ of fixed magnitude are confined to a single suriace in velocity; the differential element which the detector intercepts is denoted dS. The detector also subtends a solid angle dw in the $\mathrm{c.m}$. system; this solid angle is given by

$$
\mathrm{d} \omega=\mathrm{dS} / \mathrm{u}^{2}
$$

and

$$
\mathrm{d} \Omega=\cos (\underset{\sim}{n}, \underline{v}) \mathrm{dS} / \mathrm{v}^{2}
$$

where $\mathrm{n}_{\sim}$ is the outward normal to the surface dS, which lies along $\underset{\sim}{\text {. }}$

Equating fluxes in the c.m. and lab systems, we have

$$
I_{c \cdot m .}(\theta, u) d \omega=I_{L}(\theta, v) d \Omega
$$

or

$$
I_{L}(\theta, v)=\frac{v^{2}}{u^{2} \cos (\underline{u}, \underline{v})} I_{c \cdot m \cdot}(\theta, u) .
$$

The Jacobian for this transformation therefore differs from the previous result by the $\cos (\underline{u}, \underset{v}{\mathbf{v}})$ term originating from the fact that the scattering must be restricted to an infinitesimal shell in velocity space. Whenever this condition is no longer 
Figure 13. Newton diagram showing solid angles $d \Omega$ and $d \omega$ subtended by detector in the $1 \mathrm{ab}$ and $\mathrm{c} . \mathrm{m}$. systems. S denotes the surface mapped out by c.m. recoil vectors $\underset{\sim}{u}$ of fixed length. dS denotes the differential element of the surface intercepted by the detector, $\cong$ its outward normal. 


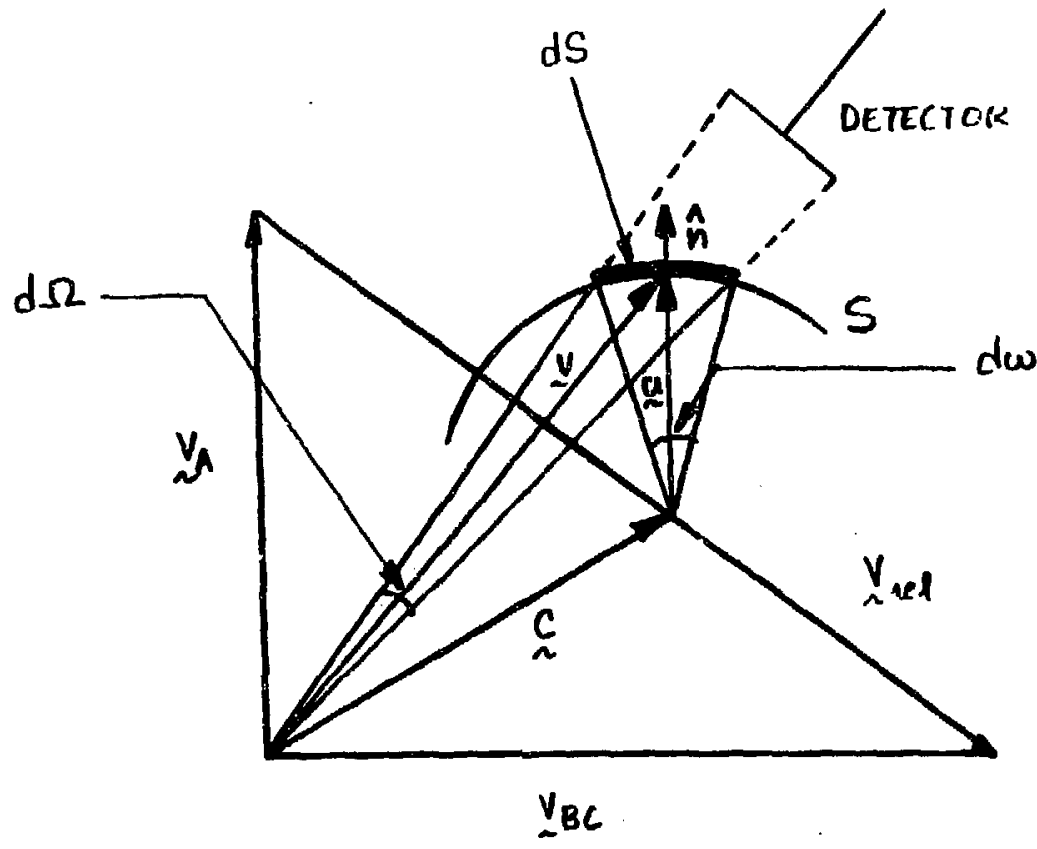

Eig. 13 
fulfilled because a distribution of states can be populated (as in reactive or multi-channel nonreactive scattering), the shell in velocity space has a finite thickness and the $\cos (\underset{\sim}{u}, \underset{\sim}{v})$ term averages over a number of $(\underset{w}{u}, \underset{v}{v})$ combinations to unity. In general, therefore, one must be extremely careful in using the above Jacobian to avoid what has been in the past a very common error.

In the general reactive case we have determined a Jacobian which relates $1 \mathrm{ab}$ and c.m. fluxes. Transforming laboratory data to the c.m. system, however, requires a knowledge of the unique $\mathrm{C}$ vector, a quantity which cannot be defined when distributions in intersection angle and beam energy exist. Deconvolution procedures ${ }^{10}$ or integration fitting routines ${ }^{11,12}$ can be applied with some success if the initial conditions are well-defined. In the latter case, in particular, reasonable interpretations of experimental data can be made by assuming a c.m. distribution in angle and recoil speed and transforming this function back to the lab while averaging over the initial conditions provided that the cross section varies weakly over the energy range of the initial conditions. An alternative method of data representation which Wolfgang and Cross $^{9}$ published and which had been used in other laboratories previously including this one, removes the distortion arising from unequal elements of volume in $1 \mathrm{ab}$ and c.m. space by determining the differential cross section for scattering products into an element $d^{3} v$ in velocity space where

$$
d^{3} y=v^{2} \sin \theta d \theta d \Phi d v
$$


As we remarked earlier, this element of volume in the lab equals the corresponding element of volume in the c.m. system. This choice contrasts with the element of volume one chooses for simultaneous scattering into a given solid angle at a given velocity, which, in the $1 \mathrm{ab}$, equals sined $\theta \mathrm{d} \phi \mathrm{dv}$. Recall that, earlier, we wrote

$$
\mathrm{d} \sigma=\mathrm{I}_{\mathrm{L}} \mathrm{dvd} \Omega=\mathrm{I}_{\mathrm{c} . \mathrm{m} .} \cdot \mathrm{dud} \omega
$$

to equate differential fluxes in the two coordinate systems. We desire a representation in which volume elements are equal, but in which we still conserve flux in the transformation between coordinate systems in relative motion. Since $d^{3} \underline{v}=v^{2} d v d \Omega$ and $d^{3} \underline{u}=u^{2} d u d \omega$, we want to find $I_{L}^{\prime}$ and $I_{c . m}^{\prime}$. such that

$$
d \sigma=I_{L}^{\prime} d^{3} y=I_{c \cdot m}^{\prime} \cdot d^{3} \underline{u}
$$

Clearly the equality of volume elements in the two systems demands that

$$
I_{L}^{\prime}=\frac{I_{L}}{v^{2}}, \quad I_{c \cdot m}^{\prime}=\frac{I_{c \cdot m} .}{u^{2}} .
$$

We call the primed representation the Cartesian representation and note that $I_{L}(\theta, v) / v^{2}$ can be generated exactly from laboratory dat: with no issumptions made on the location of the c.m. of course, the true c.m. cross section can only be extracted from the Cartesian representation by suitable averaging over $u^{2}$, so that we have not reduced the labor of extracting $I_{c . m .}(\theta, u)$ from the data, but have merely removed distortions arising 
from unequal volume elements in the different coordinate systems. A comparison of data represented in the $1 \mathrm{ab}, \mathrm{c} . \mathrm{m}$. , and Cartesian systems is presented in reference 13.

Having shown that the choice of volume element $d^{3} \mathrm{v}$ generates the Cartesian representation, we proceed with a discussion of normalization of measured intensity to the detector volume in angular and velocity space as outlined by Gentry.

Let us define the flux distribution as before and denote it $N(\theta, \Phi, v)$; the number of particles scattered into $d^{3} y$ per unit time is then

$$
\mathrm{d} C=\mathrm{N}(\theta, \Phi, \mathrm{v}) \mathrm{d}^{3} \mathrm{y}
$$

Recalling that the differential cross section $\frac{d \sigma}{d \Omega}$ is merely the ratio of the intensity scattered into rolume element d $\Omega$ to the total beam intensity, we write

$$
I(\theta, \Phi, v) d^{3} \underline{v}=\frac{N(\theta, \Phi, v) d^{3} v}{N^{0} \rho l}=\frac{d C}{N^{0} \rho l}
$$

where $\mathrm{N}^{0}$ is the number of particles per unit time incident on a target of length $\ell$ and number density $\rho$.

We must now consider the finite energy and angular bandpass of our detector; we calculate the detector volume by integrating $d^{3} y$ over the energy and angular bandpass as follows:

$$
v_{\text {det }}=\int_{\operatorname{det}} d^{3} y=\int_{\operatorname{det}} v^{2} \sin \theta \operatorname{d} \theta d \phi d v .
$$


Thus the detector measures an effective differential cross section which only convarges to the true cross section as $v_{\text {det }}$ becomes sma11. The cross section we measure is

$$
\begin{aligned}
\int_{\operatorname{det}} I(\theta, \Phi, v) d^{3} \underline{y}=\frac{C}{N^{0} \rho l} \\
\quad C=\int_{\text {det }} d C=\int_{\operatorname{det}} N(\theta, \Phi, d v) d^{3} v
\end{aligned}
$$

where $C$ defines the total number of particles striking the detector per unit time.

We can calculate the cross section such that it will converge to the time cross section as the detector resolution approaches ideality, i.e., as $v_{\text {det }}$ becomes small. We thus normalize Eq. (21) to the detector volume as follows:

$$
\bar{I}(\theta, \Phi, v)=\frac{\int_{\operatorname{det}} I(\theta, \Phi, v) d^{3} v}{\int_{\operatorname{det}} d^{3} v}=\frac{C}{N^{0} \rho l} / v \operatorname{det}
$$

We now calculate our detector volume in energy and angular space as follows: the energy bandpass of the detector is constant,

$$
\Delta E=\text { constant }
$$

the constant being determined by the analyzer geometry and the energy at which the analysis is performed. We may also evaluate the angular contribution from the solid angle subtended 
by the detector by noting that the maximum angular range $\theta_{1 / 2}$ seen by the detector is defined by a ray passing through the collision center and the detector boundary:

$$
v_{\operatorname{det}}=\int_{0}^{2 \pi} d \phi \int_{0}^{\theta} 1 / 2 \sin \theta d \theta \int_{v_{\min }}^{v_{\max }} v^{2} d v .
$$

to energy

We can change variables/in the integral over velocity and assume that the bandpass is small so that $E$ is constant in the interval $\left(E_{\min }, E_{\max }\right)$ :

$$
\begin{aligned}
& v_{\text {det }}=\int_{0}^{\theta} 1 / 2 \sin \theta d \theta \int_{E_{\min }}^{E_{\max }} E^{1 / 2} d E \\
& v_{\text {det }}=E^{1 / 2} \int_{0}^{\theta_{1} / 2} \sin \theta d \theta\left(E_{\max }-E_{\min }\right) .
\end{aligned}
$$

But $E=E_{\max }-E_{\min }$ is constant and the angular contribution can be written as $\mathrm{d}^{2} / 2 \mathrm{~L}^{2}$ where $\mathrm{d}$ is the detector aperture diameter at a distance $L$ from the collision center. Including all of the constants which have casually been ignored in the previous expressions, we have

$$
v_{\text {det }}=\frac{\pi}{4}\left(\frac{d^{2}}{L^{2}}\right)\left(\frac{2}{M}\right)^{1 / 2} k E^{1 / 2}
$$

where $k$ is the analyzer resolution constant. Thus

$$
\bar{I}(\theta, \phi, v)=\frac{4 C}{\pi N^{0} \rho l} \frac{L^{2}}{d^{2}} \frac{M^{1 / 2}}{2 E^{I / 2}}
$$

C is the intensity observed by the finite detector volume. 
The important point of this discussion is that normalizing intensities to the detector volume introduces a "Jacobian" $\mathrm{E}^{-1 / 2}$ which will weight contributions at lower laboratory energies more heavily than at higher energies. Finally, let us state again that $\overline{\mathrm{I}}(\theta, \Phi, v)$ is a Cartesian representation.

One point of importance in deriving the above result has been suppressed and requires elaboration. The calculated Cartesian density must reflect the fact that under certain circumstances, the detector does not view the entire collision center and that the fraction of the zone viewed has an angular dependence. In the beam-scattering cell arrangement, this correction is quite important since the fraction of the cell viewed is a strong function of laboratory scattering angle. In a crossed beam experiment, this factor can be made quite small and, in fact, can be eliminated if the detector aperture is larger than the maximum dimension of the collision zone. In our experimental arrangement, however, the ion beam has a profile approximately $3 \mathrm{~mm} \times 3 \mathrm{~mm}$ at the collision center whereas the detector aperture is a $1 \mathrm{~mm}$ circular aperture as required for $3 \%$ resolution for the spherical deflector.

We can make a simple estimate of the viewing factor for our geometry by considering the overlap of the detector aperture with the collision zone. We find that for a collision volume which is approximately cubic, that the leading term for the viewing function behaves 1 ike $\cos \theta$ where $\theta$ is the laboratory scattering angle. For most of the systems we have studied to date, $v_{A} \gg v_{B C}$ so the angular range over which we perform 
measurement is generally restricted to $\theta \leq 15^{\circ}$. We find therefore that the viewing factor varies from constancy by only $3.5 \%$ and in fact varies from a constant factor by less than 15 for $\theta \leq 30^{\circ}$. Consequent1y, then, our Cartesian plots portray experimental intensities as

$$
\begin{aligned}
\overline{\mathrm{I}}_{\mathrm{calc}} & =\frac{(\mathrm{S}-\mathrm{B})}{\mathrm{tE}} \ell(\theta) \\
S & =\text { signal counts in time } t \\
\mathrm{~B} & =\text { background } \\
\mathrm{E} & =1 \mathrm{ab} \text { energy } \\
\ell(\theta) & =\text { angular viewing factor. }
\end{aligned}
$$

Data reduction is effected by a Fortran program CBPLOT on the $\operatorname{CDC} 6600$ which reads input data in a reasonably convenient, format-free manner, scales the data by the appropriate energy and angular normalization factors and a Calcomp plotter generates a grid of intensities vs ( $v . \theta)$ through which smooth contours are drawn by hand. 
References, Chapter IV

1. A compjete discussion of the lab-c.m. transformation is found in E. A. Entemann, Ph.D. dissertation, Harvard University, 1967.

2. D. R. Herschbach, Adv. Chem. Phys. 10, 319 (1966).

3. E. A. Entemann and D. R. Herschbach, Faraday Discuss. Chem. Soc. $\underline{44}, 289$ (1967).

4. R. K. B. Helbing, J. Chem. Phys. 48, 472 (1968).

5. F. A. Morse and R. B. Bernstein, J. Chem. Phys. 37, 2019 (1962) .

6. T. T. Warnock and R. B. Bernstein, J. Chem. Phys. 49,1878 (1.968)

7. P. E. Siska, Ph.D. dissertation, Harvard University, 1970.

8. W. R. Gentry, UCKL-17691, Lawrence Radiation Laboratory, 1967.

9. R. Wolfgang and R. J. Cross, Jr., J. Phys. Chem. 73, 743 (1969) .

10. G. E. Ioup and B. S. Thomas, J. Chem. Phys. 46, 3959 (1967).

11. K. T. Gillen and B. H. Mahan, J. Chem. Phys, 56, 2517 (1972).

12. P. E. Siska, J. Chem. Phys. 59, 6052 (1973).

13. 2. Herman and K. Birkenshaw, Ber. Bunsenges. Phys. Chem. 77. 566 (1973). 
APPENDIX A

Listing of Mechanical Drawings

A. Molecular Beam Reaction Chamber

1. Main Vacuum Tank

$12 M 5656$

2. Rotating Detector Lid

$12 N 6236$

3. Chamber Support Plate

$12 \mathrm{M} 5804$

4. Graduated Inner Ring

$12 \mathrm{~N} 6243$

5. Bearing Retainer Ring

12M5663

6. Sprccket

$12 \mathrm{M} 5692$

7. Gate Valve

$12 \mathrm{~N} 6302$

8. Front Plate

$12 \mathrm{~N} 6311$

9. Drive Sprocket

$12 \mathrm{M} 6302$

10. Drive Sprocket Adapter

$12 \mathrm{M} 6312$

11. Flanged Tee

$12 M 5774$

12. Blind Flange

$12 \mathrm{M} 6322$

13. Chamber Window Flange

$12 \mathrm{M} 6294$

14. Chamber Support Stand

$12 \mathrm{M} 5796$

15. Jack Screw Adapter

$12 \mathrm{M} 5831$

16. Jack Screw 3/4"

$12 \mathrm{M} 5841$

17. Chamber Stand Dolly

$12 \mathrm{M} 5814$

18. 3/4" Jack Screw Bearing Pad

$12 \mathrm{M} 5821$

19. Blank Flange -1

$12 \mathrm{~N} 6991$

20. Blank Flange -2

$12 \mathrm{~N} 7001$

21. Feedthru Flange

12N7011

B. Ion Beam Focusing Syscem

1. Momentum Analyzer Chamber

$12 \mathrm{~N} 6253$

2. Momentum Analyzer Magnet

$12 \mathrm{~N} 6263$

3. Ion Source Vacuum Chamber

$12 \mathrm{~N} 6273$

4. Ion Beam Chamber

$12 \mathrm{~N} 6283$

5. Adapter Flange

$12 \mathrm{~N} 6293$

6. Initial Focusing Stage Extractor

$12 \mathrm{~N} 6601$

7. Initial Focusing Stage Element 1

$12 \mathrm{~N} 6511$

8. Initial Focusing Stage Elements 2 \& 3

9. Initial Focusing Stage Elements 4 \& 6 
10. Initial Focusing Stage Element 5

$12 N 6641$

11. Initial Focusing Stage Element 7

$12 N 6651$

12. Initial Focusing Stage Element 8

$12 N 6662$

13. Quadrupole Lens Element 9

$12 N 6671$

14. Initial Focusing Stage Element 10

$12 \mathrm{~N} 6682$

15. Initia1 Focusing Stage Support Rod

$12 \mathrm{~N} 6691$

16. Momentum Analyzer S1it

$12 \mathrm{~N} 6701$

17. Focusing Element Mounting Plate

$12 \mathrm{~N} 6712$

18. Final Focusing Stage Element 3

$12 \mathrm{~N} 6722$

19. Final Focusing Stage Elements 4 \& 6

$12 \mathrm{~N} 6732$

20. Final Focusing Stage Element 5

$12 \mathrm{~N} 6742$

21. Deflection P1ate Holder

$12 \mathrm{~N} 6751$

22. Deflection Plate

$12 \mathrm{~N} 6761$

23. Retarder Plate

$12 N 6771$

24. Retarder Entrance Slit

$12 \mathrm{~N} 6781$

25. Retarder Retainer Plate

$12 \mathrm{~N} 6791$

26. Analysis Chamber

$12 \mathrm{~N} 6803$

27. Retarder P1ate Spacer

$12 \mathrm{~N} 6901$

C. Electron Impact Ion Source

1. Filament Chamber

$12 \mathrm{~N} 7073$

2. Insulating Flange

$12 \mathrm{~N} 7082$

3. Source Body

$12 N 7092$

4. Inner Conductor

$12 N 7101$

5. Conductor Support

$12 N 7111$

6. Outer Conductor

12N7121

7. Cathode Mounting Plate

$12 N 7131$

8. Cathode Support

12N7141

9. Anode Need1e

$12 N 7151$

10. Anode Tip

$12 N 7161$

D. Molecular Beam Source

1. Chopper Mounting Block

$12 \mathrm{~N} 7022$

2. Nozzle Support Collar

$12 \mathrm{~N} 6812$

3. Nozzle llolder

$12 N 6822$

4. Beam Catcher

$12 N 6834$

5. Orifice Plate

$12 N 7031$ 
6. Alignment Fixture Support

$12 N 7043$

7. Alignment Fixture Bushing

$12 \mathrm{~N} 7061$

8. Alignment Fixture Rod

$12 \mathrm{~N} 7051$

9. Mounting Plate

$12 \mathrm{M} 8882$

10. Collector Flange

$12 \mathrm{M} 8893$

11. Collector Pillar

$12 \mathrm{M} 8901$

E. Energy Analyzer

1. Analyzer Mount

$12 \mathrm{~N} 6473$

2. Convex Electrode

$12 \mathrm{~N} 6452$

3. Concave Electrode

$12 \mathrm{~N} 6432$

4. Concave Electrode Holder

$12 \mathrm{~N} 6442$

5. Shielding Box

$12 N 6512$

6. Shielding Plate

$12 \mathrm{~N} 6522$

7. Electrode Mounting Plate - 1

$12 \mathrm{~N} 6561$

8. Electrode Mounting Plate - 2

$12 \mathrm{~N} 6571$

9. Electrode Mounting Plate -3

$12 \mathrm{~N} 6581$

10. Pre-analyzer Lens Mount

$12 \mathrm{~N} 6462$

11. Detector Aperture

12 N6531

12. Read Lens Elements 1,2

$12 \mathrm{~N} 6541$

13. Read Lens Element 3

$12 \mathrm{~N} 6551$

14. Field Leas Elements $A$ and $B$

$12 \mathrm{~N} 6591$

15. Post Analyzer Lens Holder

$12 N 6502$

16. Post Analyzer Ion Lens 1

$12 N 6481$

17. Post Analyzer lon Lens 2,3

$12 \mathrm{~N} 6491$

F. Quadrupole Mass_Spectrometer

1. Entrance Lens

12 N6961

2. Exit Lens

$12 N 6971$

3. QPMS Pole Rod

$12 \mathrm{M} 5982$

4. End Insulator 2

$12 \mathrm{M} 6003$

5. Find Insulator 3

$12 \mathrm{M} 6753$

6. QPMS End Insulator Shield

$12 \mathrm{M} 6013$

7. 4PMS Plain Washer

$11 \mathrm{J8952}$

8, 4PMS Pigtail Washer 
G. Ion Detector

1. Detection Chamber

$12 M 8464$

2. Cathode Flange

$12 \mathrm{M} 8473$

3. High Voltage Protection Spool

$12 \mathrm{M} 9512$

4. High Voltage Protection Shield

$12 \mathrm{M} 9523$

5. Sliding Seal

$12 \mathrm{M} 8391$

6. Kovar Screw

$12 \mathrm{M} 8381$

7. Ion Target

$12 \mathrm{M} 8401$

8. Shielding Sleeve

$12 \mathrm{M} 9501$

9. Anti-corona Ball

$12 \mathrm{~N} 6381$

10. QPMS Chamber

$12 \mathrm{~N} 6323$

11. Ion Lens Holder

$12 \mathrm{~N} 6342$

12. Ion Lens 2 \& 3

$12 \mathrm{~N} 6371$

13. Window Lens

$12 \mathrm{~N} 6361$

14. Port Hole Cover

$14 J 9722$

15. High Voltage Connector Adaptor

$14 \mathrm{~K} 3032$

EMI 9824B PMT Accessories

16. PMT Chamber

$12 N 6352$

17. PMT Housing

$12 N 6332$

18. PMT Housing Endcap

$12 \mathrm{~N} 6421$

19. PMT Scintillator Mounting Nut

$12 \mathrm{~N} 6401$

20. Dynode Chain Holder

$12 \mathrm{~N} 6411$ RCA 8575 PMT Accessories

21. PMT Chamber - 2

$12 \mathrm{~N} 6913$

22. PMT Liousing -2

$12 \mathrm{~N} 6922$

23. PMT Housing Endcap

$12 \mathrm{~N} 6931$

24. Feedthru Mounting Ring

14K1513-2

25. PMT Scintillator Mounting Nut -2

$12 \mathrm{~N} 6941$

26. Dynode Chain Holder -2

$12 \mathrm{~N} 6951$

H. Assemb1y Drawings

1. Reaction Chamber Assembly

$12 \mathrm{~N} 6844$

2. Scintillation Detector

$12 N 6854$

3. Energy Analyzer

$12 N 6864$

4. Ion Source Mass Spectrometer

$12 \mathrm{~N} 6873$

5. Initial Focusing Stage

$12 \mathrm{~N} 6883$

6. Final Focusing Stage

$12 \mathrm{~N} 6893$

7. Electron Impact Source 
APPENDIX B

List of Electronic Prints

A. Vacuum System

1. Standard Ion Gauge Power Supplies

5Z1814-9

LE12283-1M2

2. IMR Diffusion Pump Power Interlock

851764 Mod.2

3. Hastings Dual Vacuum Interlock

624664

4. Hastings Dual Vacuum Gauge

623842

5. Ion Gauge Switching Pane1

852781

6. DP Control Panel

852741

7. Single Vacuum Control

$4 \times 7033$

8. Baker System Wiring Diagram

8S2925A

B. Experimenta1 Instrumentation

1. Ion Source Filament Power Supply

953802

2. $30 \mathrm{KV}$ DC Power Supply

$17 \times 4034$

3. Quadrupole Mass Filter Supply

$8 \mathrm{~S} 3234-1$

4. Quadrupole Mass Filter Rectifier

855843

5. Ion Beam Focusing Control

$7 S 1684$

6. Dual Channel Gate-Gating and Timing Logic

$7 S 1694$

7. Dual Channel Gate - Power Supply and Pulser

Board $7 \mathrm{~S} 1702$

8. $150 \mathrm{~Hz}$ Tuned Amplifier

$7 \mathrm{~S} 1712$ 